\title{
A Lagrangian Study of Interfaces at the Edges of Cumulus Clouds
}

\author{
Vishnu NAIR, ${ }^{\mathrm{a}}$ ThIJs Heus, ${ }^{\mathrm{b}}$ AND MAARTEN VAN REEUWIJK ${ }^{\mathrm{a}}$ \\ ${ }^{a}$ Department of Civil and Environmental Engineering, Imperial College London, London, United Kingdom \\ ${ }^{\mathrm{b}}$ Department of Physics, Cleveland State University, Cleveland, Ohio
}

(Manuscript received 3 June 2020, in final form 13 April 2021)

\begin{abstract}
Interfaces at the edge of an idealized, nonprecipitating, warm cloud are studied using direct numerical simulation (DNS) complemented with a Lagrangian particle tracking routine. Once a shell has formed, four zones can be distinguished: the cloud core, visible shell, invisible shell, and the environment. The union of the visible and invisible regions is the shell commonly referred to in literature. The boundary between the invisible shell and the environment is the turbulent-nonturbulent interface (TNTI), which is typically not considered in cloud studies. Three million particles were seeded homogeneously across the domain and properties were recorded along individual trajectories. The results demonstrate that the traditional cloud boundary (separating cloudy and noncloudy regions using thresholds applied on liquid condensate or updraft velocity) are some distance away from the TNTI. Furthermore, there is no dynamic difference between the traditional liquid-condensate boundary and the region extending to the TNTI. However, particles crossing the TNTI exhibit a sharp jump in enstrophy and a smooth increase in buoyancy. The traditional cloud boundary coincides with the location of minimum buoyancy in the shell. The shell premixes the entraining and detraining air and analysis reveals a highly skewed picture of entrainment and detrainment at the traditional cloud boundary. A preferential entrainment of particles with velocity and specific humidity higher than the mean values in the shell is observed. Large-eddy simulation of a more realistic setup detects an interface with similar properties using the same thresholds as in the DNS, indicating that the DNS results extrapolate beyond their idealized conditions.
\end{abstract}

KEYWORDS: Buoyancy; Convective clouds; Entrainment; Lagrangian circulation/transport; Turbulence; Updrafts/downdrafts

\section{Introduction}

Cumulus convection is one of the most important unresolved processes in weather and climate forecasting. The parameterization of different processes in convective schemes such as the representation of turbulent fluxes is a major source of uncertainty in numerical weather prediction models and global circulation models (Bony et al. 2015). Some of the most successful convective parameterizations employ massflux schemes (bulk and spectral). In such schemes a cloud model is employed to specify the upward mass flux through the cloud base. These mass flux schemes are mostly of two types: bulk schemes where a single entraining-detraining plume represents the combined effect of an ensemble of active cumuli, or spectral schemes where multiple plumes are considered. Some examples (not an exhaustive list by any means) of the bulk mass flux models are the schemes by Tiedtke (1989), Kain and Fritsch (1990), Bechtold et al. (2001), Gregory and Rowntree (1990), and Gerard (2015), and some popular spectral methods are the works of Arakawa and Schubert (1974), Johnson (1976), and Moorthi and Suarez (1992). Two important parameters that modify the mass flux and the vertical transport of temperature, moisture and momentum in these models are the entrainment and detrainment rates. A multitude of parameterizations exist to calculate these rates as summarized in de Rooy et al. (2013). Most of the schemes mentioned so far calculate the change of mass flux with height using entrainment and detrainment rates, which have been

Corresponding address: Vishnu Nair, vs2016@imperial.ac.uk divided into large-scale dynamical rates and smaller-scale turbulent rates.

LES has become the primary tool to diagnose entrainment and detrainment rates and it has served the community well over the past years with the majority of the parameterizations arising from LES studies (de Rooy et al. 2013, and references therein). A traditional definition to diagnose bulk entrainment and detrainment rates through LES is (Siebesma 1998)

$$
\begin{aligned}
& E_{\phi}=-\frac{1}{A \phi_{e}} \oint_{\partial \Omega_{E}} \rho \hat{\mathbf{n}} \cdot\left(\mathbf{u}-\mathbf{u}_{\mathbf{i}}\right) \phi d l, \\
& D_{\phi}=\frac{1}{A \phi_{c}} \oint_{\partial \Omega_{D}} \rho \hat{\mathbf{n}} \cdot\left(\mathbf{u}-\mathbf{u}_{\mathbf{i}}\right) \phi d l,
\end{aligned}
$$

where $E_{\phi}$ and $D_{\phi}$ are the entrainment and detrainment rates, $\phi$ is a conserved scalar, and the subscripts $c$ and $e$ denote averages over the cloud and environment, respectively. The path integral is taken horizontally over a cloud area $A$ at a constant vertical height. Here the cloud boundary $\partial \Omega$ has been decomposed into an entraining and detraining region, denoted $\partial \Omega_{E}$ and $\partial \Omega_{D}$, respectively. The regions are defined by the sign of the net entrainment velocity across the interface $\hat{\mathbf{n}} \cdot\left(\mathbf{u}-\mathbf{u}_{\mathbf{i}}\right)$, and clearly $\partial \Omega=\partial \Omega_{E} \cup \partial \Omega_{D}$.

Although the definitions above are rigorous, the entrainment/detrainment rates will depend on the choice of the interface $\partial \Omega$. There are multiple interfaces that can be chosen. Most operational parameterizations use the boundary of the cloud core as the interface over which entrainment is calculated. Different definitions of the cloud core interface exist: often these are based on nonzero threshold values on liquid 
water content and vertical velocity (Romps 2010) or thresholds on buoyancy and/or vertical velocity (de Roode and Bretherton 2003). Convective structures can also be identified using decaying passive tracers that are constantly emitted from the surface (Couvreux et al. 2010; Park et al. 2016, 2017). Siebesma and Cuijpers (1995) approximated fractional entrainment and detrainment rates using LES and evaluated the effect of different definitions for the cloudy area. They employ a single bulk active cloudy part and a passive environmental part and define the active cloud domain in different ways: cloud (all grid cells with nonzero liquid water), updraft (nonzero liquid water with positive vertical velocity), and core (nonzero liquid water, positive vertical velocity, and positive buoyancy). Their study reveals that the updraft sampling gives the best approximations for the turbulent fluxes of the conserved variables $\theta_{l}$ and $q_{t}$ (for the temperature and humidity, respectively). This is because the updraft sampling includes the negatively buoyant but decelerating part of the updraft rise as well. A different decomposition (as originally proposed by Tiedtke 1989) into an updraft, a downdraft and an environment was also evaluated to check the role of downdrafts. This did not prove to be better than the updraft sampling but it was stated that this would be relevant in the cases where the mass flux in the downdraft would be comparable to that in the cloud updraft. A more recent study by Gu et al. (2020) has shown that a bulk mass flux parameterization using a simple cloud-environment decomposition does not capture the correct magnitude and vertical transport of turbulent fluxes of heat and humidity. Inclusion of the downdraft significantly improved mass flux parameterizations (especially at the cloud top).

Romps (2010) recognizes the importance of defining the interface over which entrainment takes place and defines two categories, active and inactive, whereby a Lagrangian parcel can be considered to have entrained when it flips from inactive to active and vice versa for detrainment. The atmosphere is divided into these two categories using thresholds for a condensate mixing ratio and the vertical velocity. The "local" rates calculated in Romps (2010) revealed values twice as high as that in the particle budget calculations in bulk plume schemes. Dawe and Austin (2011) investigated the difference between the bulk (from bulk mass flux schemes) and local rates, and attributed this to the presence of the moist and negatively buoyant subsiding shells around the cloud cores and drier air at the edge of the cloud core. Bulk plume mass flux parameterizations define the properties of entrained air as the horizontal slab average over the environment and that of the detrained air as the slab average over the cloud core as shown in Eqs. (1a) and (1b). However, the presence of the shell means that the air entering and leaving the cloud is effectively premixed.

As explained in de Rooy et al. (2013), it is important to note that the two approaches will result in the correct turbulent transport as long as the mixing coefficients $\varepsilon$ and $\delta$ are diagnosed and applied in the relevant framework correctly. Smaller $\varepsilon(\delta)$ values combined with the higher difference between the entraining (detraining) air and the cloud (environment) in the bulk approach should result in the same lateral turbulent flux as in the direct approach. Dawe and Austin (2011) also showed a preferential entrainment of parcels with average humidity and vertical velocity higher than that of the mean shell properties (thus enhancing the flux transfer). This makes it highly relevant to take a closer look at a cloud edge and focus on what effect the choice of interface has on air parcels at the cloud edge. This is the primary motivation for the present work.

A detailed study of lateral mixing at the cloud edge and the dynamics of the shell was performed by Nair et al. (2020, hereafter NHvR20) using direct numerical simulation (DNS). Cloud edge studies using DNS are performed at moderate Reynolds numbers and consider idealized setups, often using mixing layers as models for the cloud edge. One of the main drawbacks of such mixing layer models is that the shell layer exhausts the cloud layer very rapidly and a very transient negatively buoyant layer is formed, which grows at the expense of the cloud layer. In NHvR20, a canonical setup was developed, which solves this problem by applying a forcing term over a positively buoyant cloud layer, which nudges cloud properties to predefined values. This ensures the presence of an actively growing cloud throughout the simulation and such a numerical setup is well suited to look at cloud edge interfaces. Furthermore, the entrainment coefficient calculated in NHvR20 was on the same order of magnitude as those calculated in LES studies (Yeo and Romps 2012). This suggests that DNS can play an important role as a tool to study flow at cloud edges. The DNS results for the buoyancy distribution in NHvR20 revealed the different possible interfaces over which cloudenvironment mixing can occur. The formation of the shell gives rise to two interfaces, which could possibly be of interest. The interface separating regions with zero and nonzero liquid water lies within the shell and this is the first interface of interest. NHvR20 showed that this interface is at the point where the buoyancy in the shell is a minimum. We shall refer to this interface as the visible shell boundary (VSB) since it separates the visible part of the shell (which has nonzero liquid water) from the invisible region of the shell (no liquid water). The second interface of interest is the outer boundary of the shell, which separates the turbulent region at the cloud edge from the nonturbulent environment, i.e., the turbulent-nonturbulent interface (TNTI). The TNTI has been extensively studied at the edges of jets, plumes, wakes, mixing layers and boundary layers as reviewed in da Silva et al. (2014). The interface layer is known to include two adjacent layers, the viscous super layer (VSL) where vorticity is introduced through diffusion, and the turbulent sublayer (TSL), which matches the vorticity from the turbulent region to the VSL. The TNTI is considered to be a surface with zero thickness between or within these two layers. However, this interface has been rarely considered in most cloud-edge studies. LES studies using a decaying scalar emitted from the surface to identify coherent structures in the convective boundary layer (Couvreux et al. 2010; Park et al. 2017) report areas around the cloud with elevated levels of those scalars, quickly dropping off when moving further away into the environment. It is possible that these areas are a proxy for the TNTI. In this paper we will show that, as far as this idealized flow is concerned, the TNTI represents the outer edge of the cloud system. 
The effectiveness of Lagrangian particle tracking in clouds was demonstrated by Heus et al. (2008), who used LES and tracked massless Lagrangian particles that followed the flow to study mixing between clouds and the environment. This study settled the debate surrounding the origin of in-cloud air by tracing back cloud-air parcels, clearly revealing the absence of significant cloud-top mixing and that practically all mixing occurs laterally. It is also worth mentioning that in the atmosphere, detrainment layers are often associated with locally increased stratification (de Rooy et al. 2013). Yeo and Romps (2012) used Lagrangian particle tracking on an individual cumulus cloud and calculated a higher rate of entrainment compared to the Eulerian direct measurements. This was attributed to the fast recirculation of air in and out of the core. It was also shown that almost half of the air entrained by a cloud during its lifetime had been previously detrained. These were effects that could not be captured or resolved by Eulerian measurements.

The combination of DNS and a Lagrangian particle tracking routine facilitates a study of mixing and entrainment over the different interfaces at cloud edges in detail. We will highlight the importance of the TNTI and hence the importance of including the shell in cloud edge mixing studies. By following individual particles as they entrain and detrain across the different interfaces, we show that a Lagrangian parcel can be considered to be "entrained" when it crosses the TNTI and that the shell extends the cloud edge to this interface. We also aim to highlight the degree of premixing done by the shell to the entraining and detraining air parcels. The case setup of the DNS is intentionally designed to avoid the complexity of a real cloud and only focus on the process of lateral mixing and entrainment at the cloud edge. To investigate the extent to which the findings are transferable to realistic clouds, we perform large-eddy simulation (LES) of a BOMEX case with no mean wind, and explore whether we can detect the TNTI there. Using conditional averages of the enstrophy and mass flux we show that the different interfaces can be distinctly identified and present results highlighting the net mass exchange between the different zones.

\section{Case setup and simulation details}

\section{a. Direct numerical simulations}

The case is identical to that used in NHvR20 and is shown in Fig. 1. The domain is divided into a cloud and environmental layer. The cloud layer is moist and positively buoyant and the environment is dry and its buoyancy is defined to be zero. As in Abma et al. (2013), the dominant mixing is assumed to occur locally and hence the influence of the cloud top and base can be neglected which makes the system statistically homogeneous in the vertical direction $\hat{z}$. This allows us to impose periodic boundary conditions on the top and bottom boundaries $(\hat{z}$ direction) and in the spanwise direction ( $\hat{y}$ direction) if the domain is large enough. Free-slip boundary conditions are imposed along the $\hat{x}$ direction. To prevent the evaporation of the cloud due to turbulent mixing, a forcing is applied over the initial cloud layer from $x=0$ to $x=1 \mathrm{~m}$ to nudge the values of

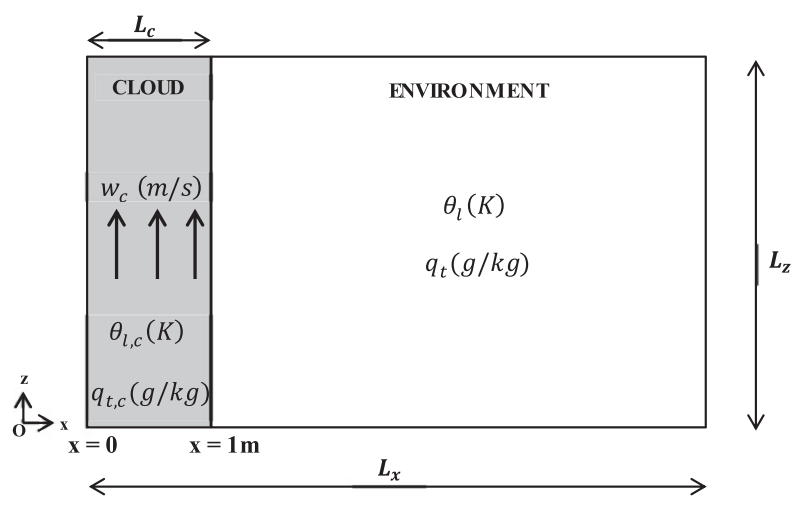

FIG. 1. Numerical setup. A volumetric forcing is applied over the gray cloud region from $x=0$ to $x=1 \mathrm{~m}$.

the vertical velocity $w$, the temperature $\theta_{l}$ and humidity $q_{t}$ to predefined values $w_{c}, \theta_{l, c}$, and $q_{t, c}$, respectively. Details of the forcing scheme can be found in NHvR20. A negatively buoyant shell forms due to the nudging in the cloud layer, which then develops in a self-similar fashion. Two distinct flow phases were observed in NHvR20 within a negatively buoyant turbulent cloud-environment mixture. The first is a "drag" phase where the momentum flux transfer (between the cloud core and the shell) dominates and the negatively buoyant shell is dragged vertically upward by the active cloud layer. The onset of the second "buoyancy" phase occurs when the negative buoyancy within the shell overcomes the drag and consequently the shell starts descending. Another pertinent finding in NHvR20 is that the shell falls ballistically and the mean velocity inside the shell is dynamically unimportant.

The code for direct numerical simulation, SPARKLE, solves the incompressible Navier-Stokes equations under the Boussinesq approximation, and transport equations for scalars to fourth-order accuracy. The buoyancy $b$ is given by

$$
b=g\left[\frac{\theta-\theta_{0}}{\theta_{0}}-\left(1-\frac{R_{v}}{R_{d}}\right)\left(q_{t}-q_{t, 0}\right)-\frac{R_{v}}{R_{d}}\left(q_{l}-q_{l, 0}\right)\right],
$$

where $\theta$ is the potential temperature; $q_{l}$ is the liquid water specific humidity; $\theta_{0}, q_{l, 0}$, and $q_{t, 0}$ are the environmental values of the potential temperature, liquid water specific humidity, and total water specific humidity, respectively; and $R_{d}=$ $287.0 \mathrm{~J} \mathrm{~kg}^{-1} \mathrm{~K}^{-1}$ and $R_{v}=461.5 \mathrm{~J} \mathrm{~kg}^{-1} \mathrm{~K}^{-1}$ are the gas constants for dry air and water vapor, respectively. The value of $q_{l, 0}$ is set to zero. A bulk condensation scheme developed by Sommeria and Deardorff (1976) is used to diagnostically calculate $q_{l}$ in the cloud layer. Further details of the numerical method used in SPARKLE can be found in Craske and van Reeuwijk (2015).

The simulation is performed on a domain of $30 \mathrm{~m} \times 15 \mathrm{~m} \times$ $15 \mathrm{~m}$ using a grid of $1536 \times 768 \times 768$ points. The initial profiles are the same as simulation A03 in NHvR20 and the simulation is run for $80 \mathrm{~s}$. The liquid water specific humidity and vertical velocity in the cloud is $q_{l, c}=3 \mathrm{~g} \mathrm{~kg}^{-1}$ and $w_{c}=0.81 \mathrm{~m} \mathrm{~s}^{-1}$, respectively. The resulting cloud buoyancy is $b_{c}=0.046 \mathrm{~m} \mathrm{~s}^{-2}$. A top-hat profile is implemented for $\theta_{l}$ and $q_{t}$ with $\Delta \theta_{l}=-5.9 \mathrm{~K}$ and $\Delta q_{t}=5.5 \mathrm{~g} \mathrm{~kg}^{-1}$. The kinematic viscosity $\nu$ is 
$4 \times 10^{-4} \mathrm{~m}^{2} \mathrm{~s}^{-1}$. The Taylor-Reynolds number $\left(\operatorname{Re}_{\lambda}\right)$ at the end of the simulation is 91 and the grid resolution $r=\Delta x / \eta=$ 1.3 where $\eta$ is the Kolmogorov length scale. The integral shell time scale for the simulation $\tau=l_{e} / u^{\prime}$ is $14.4 \mathrm{~s}$ and the Kolmogorov time scale $t_{\eta}=(v / \varepsilon)^{0.5}$ is $0.45 \mathrm{~s}$ (where the integral length scale $\left.l_{e}=u^{\prime 3} / \varepsilon\right)$. Here $u^{\prime}$ is the rms velocity and $\varepsilon$ is the dissipation rate. It is important to mention here that $\varepsilon$ is calculated as an average over the width of the shell and not the entire cloud domain. Hence the time scales $\tau$ and $t_{\eta}$ are representative of the characteristic time scales of the largest and smallest eddies inside the shell.

A Lagrangian particle tracking routine has been implemented to solve the equation of motion for massless particles:

$$
\frac{d \mathbf{x}}{d t}=\mathbf{u}(\mathbf{x}, t)
$$

Here, $\mathbf{u}=(u, v, w)$ is the velocity vector with $u, v$, and $w$ as the horizontal, transverse, and vertical components, respectively, and $\mathbf{x}=(x, y, z)$ is the particle position vector. A tricubic Hermite interpolation scheme is used to calculate the particle velocities from the Eulerian flow velocity fields. A third-order Adams-Bashforth time-stepping scheme is used to integrate the particle locations at each time step. SPARKLE uses a twodimensional domain decomposition and is parallelized using MPI. The particles on processor boundaries are communicated across processors after each time integration step following the flow field (Perrin and Jonker 2015).

A total of 3 million particles are seeded into the numerical domain after the flow has reached a self-similar state at $t_{0}=$ $68 \mathrm{~s}$. Importantly, by this stage, the flow is in the buoyancy regime and there is a descending shell present in the domain (at earlier times, the shell is only visible in the turbulence but the cloud boundary is still dragged up due to the turbulence; see NHvR20). Figure 2 shows the mean buoyancy and vertical velocity profiles at the time of seeding. The profiles are normalized by their respective minimum values $b^{*}$ and $w^{*}$. The particles are seeded homogeneously along the $x, y$, and $z$ axes within a volume bounded by $[0,9] \mathrm{m},[0,15] \mathrm{m}$, and $[0,30] \mathrm{m}$, respectively. Since the outer boundary of the shell is at approximately $7.5 \mathrm{~m}$ at the end of the simulation, the particles are seeded only up to a distance of $9 \mathrm{~m}$ across the $x$ axis. The region from $9 \mathrm{~m}$ to the boundary at $15 \mathrm{~m}$ is the quiescent environment and the results are not affected by not seeding within this region. Data for the particles are written every $0.2 \mathrm{~s}$.

Since the flow in the shell has been shown to be self-similar in NHvR20, we expect the results to hold for different initial values/parameters. The sensitivity of the results is analyzed in appendix B by performing a simulation with different initial parameters (simulation A06 in NHvR20), and the plots reveal that the particle behavior is independent of the initial flow parameters.

\section{b. Large-eddy simulations}

To explore the feasibility of an interface in more realistic set ups, we use the MicroHH LES (van Heerwaarden et al. 2017) to run an LES of shallow cumulus convection based on the BOMEX case (Siebesma et al. 2003) at an isotropic $4.1 \mathrm{~m}$ grid spacing, using $800 \times 800 \times 800$ grid points and a $3.2 \mathrm{~km}$
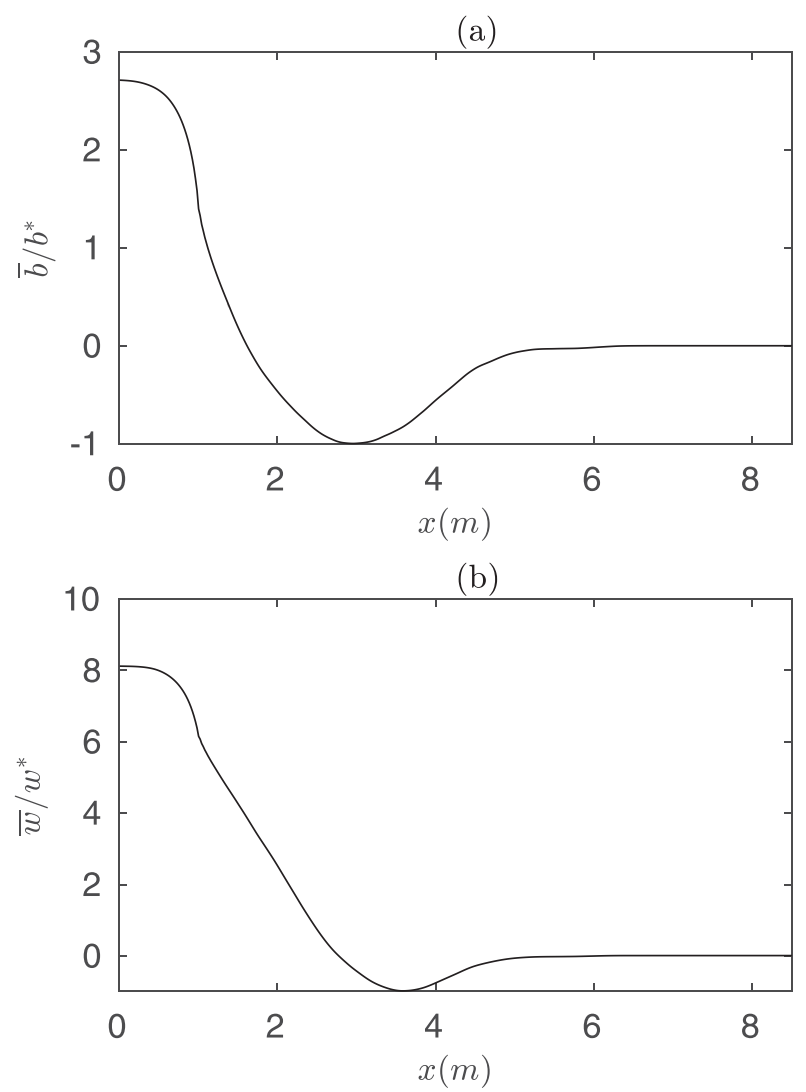

FIG. 2. (a) Mean buoyancy $\bar{b}$ and (b) mean vertical velocity $\bar{w}$ normalized by their respective minimum values $b^{*}$ and $w^{*}$ at $t_{0}$ when particles are seeded.

horizontal domain size. Note that this grid spacing is much finer than the $25 \mathrm{~m}$ that Heus and Jonker (2008) found was sufficient for a converging mass flux in the subsiding shell. With this resolution, the internal dynamics of the shell are better resolved and the enstrophy approaches a meaningful value. We use a simulation time of $10 \mathrm{~h}$, of which the first $3 \mathrm{~h}$ are discarded as spinup. To separate shear driven turbulence from convective turbulence, we set the geostrophic wind to zero. As it turns out, shear does not seem to make a major difference to our results, other than enlarging the shell size.

\section{Interfaces at the cloud edge}

As mentioned in the introduction, multiple interfaces exist at the cloud edge over which we can consider entrainment and detrainment. In this section we clearly define and visualize the two interfaces that exist due to the presence of the shell, i.e., the VSB and the TNTI. We also include the cloud core boundary (CCB), which is commonly used in most parameterization studies. The CCB and the VSB are defined by applying thresholds on the buoyancy $b$ and the liquid water specific humidity $q_{l}$. The TNTI is defined by applying a threshold on the enstrophy $\omega^{2}$. The enstrophy is a scalar quantity, which is defined as $\omega^{2}=\boldsymbol{\omega} \cdot \boldsymbol{\omega}$, where $\boldsymbol{\omega}=\nabla \times \mathbf{u}$ is the 
vorticity. We apply a threshold value of $q_{l, \text { th }}=10^{-5} \mathrm{~kg} \mathrm{~kg}^{-1}$ and $\omega_{\mathrm{th}}^{2}=10^{-6} \mathrm{~s}^{-2}$. An analysis on the sensitivity of the TNTI location to the choice of enstrophy threshold value is performed in appendix A. The three interfaces divide the domain into four different zones, which can be defined as

1) cloud core (CC), where $b>0, q_{l} \geq q_{l, \mathrm{th}}$;

2) visible shell (VS), where $b<0, q_{l} \geq q_{l, \mathrm{th}}$;

3) invisible shell (IS), where $b<0, q_{l}<q_{l \text {,th }}, \omega^{2} \geq \omega_{\mathrm{th}}^{2}$; and

4) environment (E), where $b=0$ and $\omega^{2}<\omega_{\mathrm{th}}^{2}$.

These zones are shown in Fig. 3, depicting the CC (red), VS (blue), IS (yellow), and the $\mathrm{E}$ (white). The CCB is defined on the interface between the CC and the VS, the VSB on the interface between the VS and the IS, and the TNTI on the interface between the IS and E. The snapshot represents the flow at $t_{0}$. For reference, the boundary considered by Romps (2010) would be closer to the CCB than the VSB due to the high threshold applied to the vertical velocity in that study.

Interestingly, the VSB coincides with the location where the buoyancy is minimum. Evaporation of cloud liquid water due to mixing and entrainment results in latent heat absorption. This evaporative cooling is maximum at the point where all the liquid water has evaporated, i.e., at the VSB. This can be explained using Eq. (2). When $q_{l}$ drops to zero due to complete evaporation, the consequent temperature drop results in a negative value for $\left(\theta-\theta_{0}\right) / \theta_{0}$ with the buoyancy falling to a minimum (NHvR20).

In the rest of the manuscript, all references to the "shell" indicate a union/combination of both the IS and the VS.

\section{Lagrangian particles}

Lagrangian particles are seeded uniformly across the four zones at $t=t_{0}$ over the interval $0<x<9,0<y<15,0<z<$ $30 \mathrm{~m}$. A total of 3 million particles were divided among the different zones with 578964 in the CC, 502801 in the VS, 347348 in the IS and 1572052 in the E. Since the particles are passive, they follow the flow. A snapshot of the particles at a normalized time $t^{*}=\left(t-t_{0}\right) / \tau=0.67$ is shown in Fig. 4 . The particles are colored according to the zones in which they are initially seeded at $t_{0}$ : CC (red), VS (blue), IS (yellow), and E (pink). This figure provides a qualitative idea of the source and destination of entraining and detraining particles. The CC contains particles from the VS and IS, there are indications of $\mathrm{CC}$ particles in the IS and even indications of $\mathrm{E}$ particles in the VS.

Figure 5 quantifies the movement of the particles between the zones by showing an origin-destination matrix for the initial and final zones of all particles that leave their zone of origin. Each square in the matrix represents a particular zone. The rows represent the zone of origin and the columns represent the destination zone at $t^{*}=0.67$. The colors (and the numbers) indicate the percentage of particles that originated in the zone represented by the row and has a final destination in the zone represented by the column. The percentages are calculated over the total number of particles (in all four zones) at $t^{*}$. Hence the sum of values in all the cells in the matrix is equal to 100 . It is necessary to exercise caution when

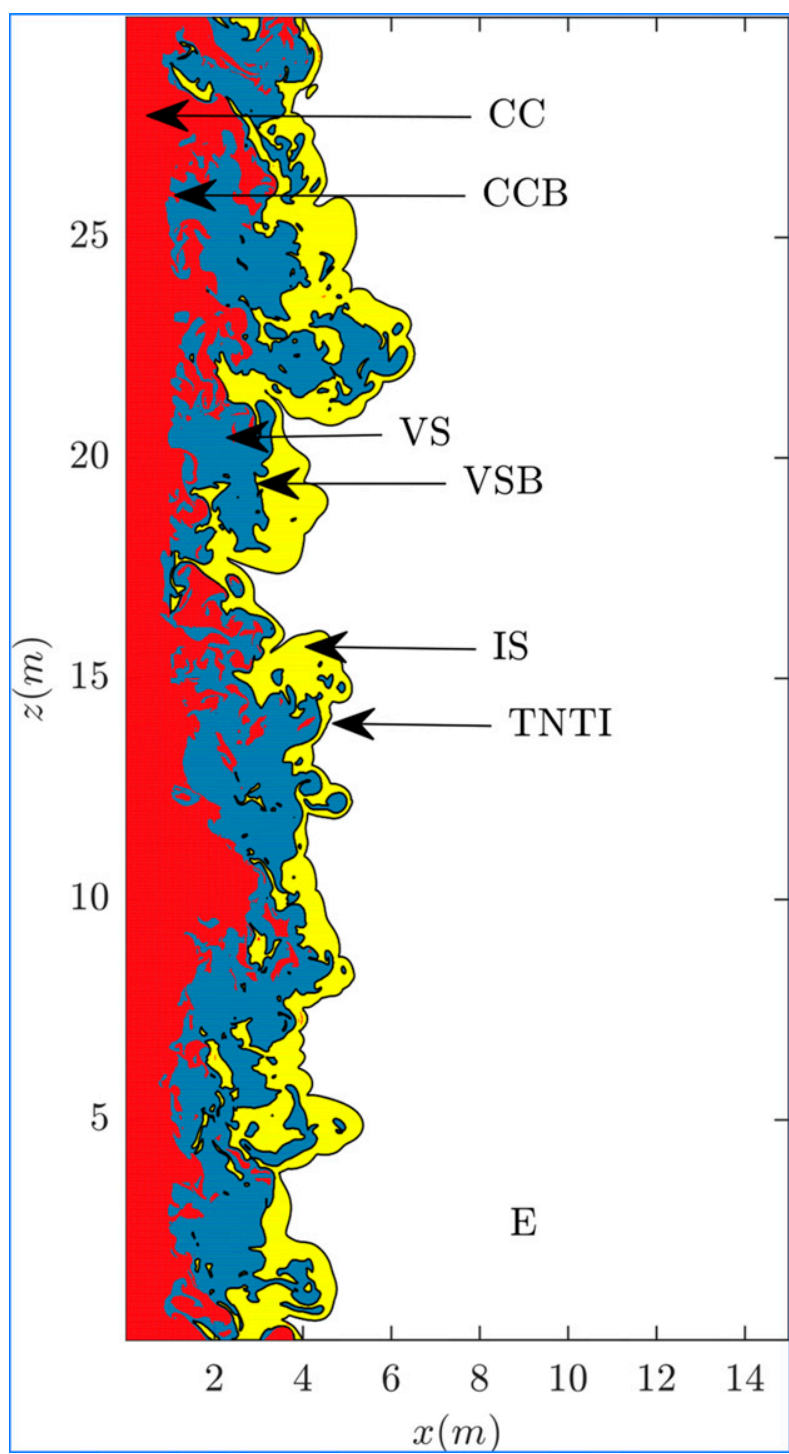

FIG. 3. The different zones at the edge of the cloudy domain at the time of seeding $\left(t_{0}\right)$. The color scheme is red-cloud core (CC); blue-visible shell (VS); yellow-invisible shell (IS); and white-environment (E). Also shown are the interfaces: cloud core boundary (CCB), visible shell boundary (VSB), and turbulentnonturbulent interface (TNTI).

generating such a matrix since the percentages will be dependent on the initial seeding density and the domain size. For instance, an analysis of the particles that started and finished in their zones of origin revealed that a high percentage simply do not make any crossing at all. In the time interval from $t^{*}=0$ to 0.67 , the following percentage (number) of particles remain in their zones of origin without making any crossing: CC-89\% (385 146); VS-73\% (221 989); IS—90\% (163162); and $\mathrm{E}-99.9 \%$ (1380 563). Hence all particles that remain in their zones of origin are excluded to give a better understanding of mixing and crossing between different zones. We still have a significant number of particles to obtain reliable 


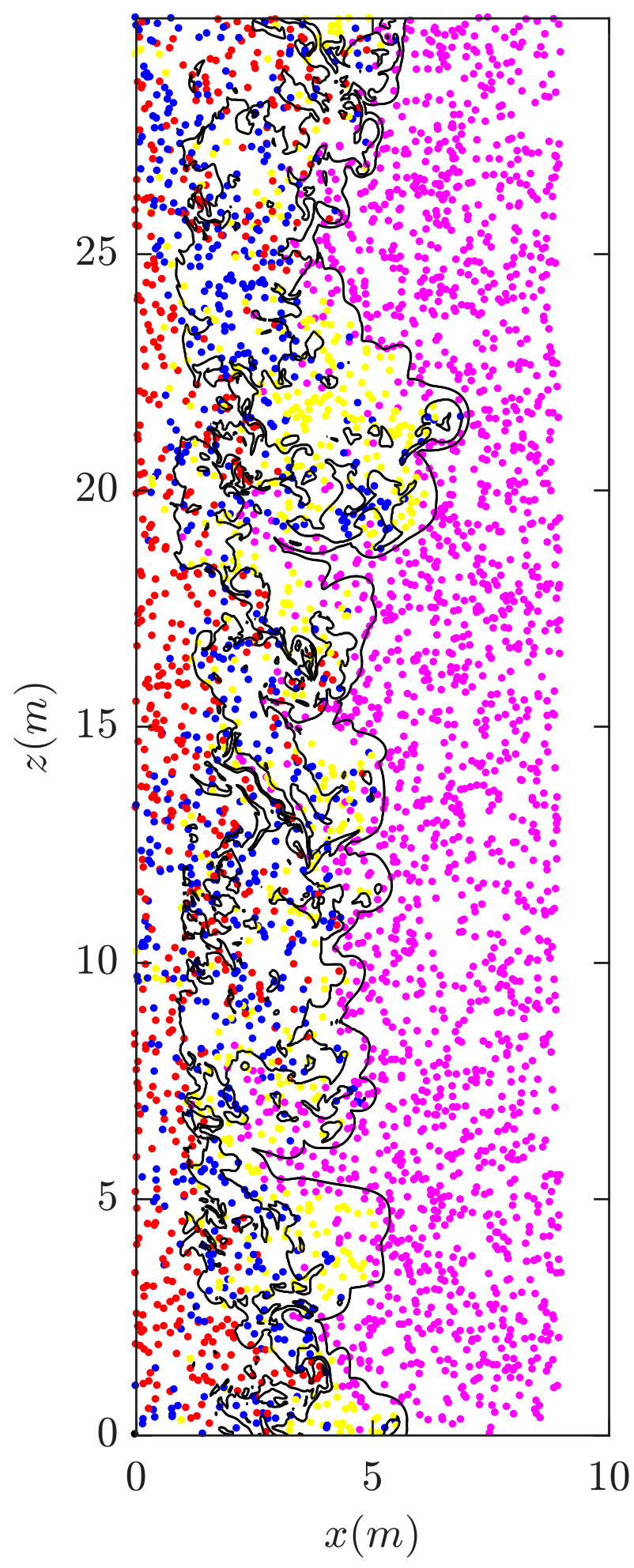

FIG. 4. Instantaneous particle positions on a two-dimensional slice of the flow $t^{*}=0.67$. Also shown are the three interfaces. Particle colors represent the zone in which they were initially seeded: red-CC; blue_-VS; yellow—IS; and pink-E.

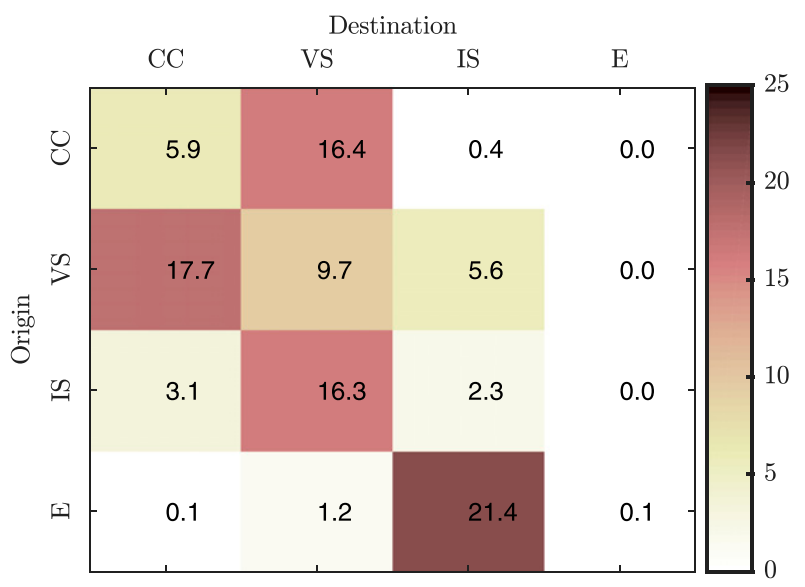

FIG. 5. An origin-destination matrix for the initial and final zones of all particles that leave their zone of origin. Colors show the percentage-wise distribution of particles based on the zone of origin at the time of seeding and the zone where they end up at time $t^{*}=0.67$.

statistics for Fig. 5: CC-193 818; VS-280 812; IS-184 186; and E-191489.

Particles starting in the CC detrain into the VS $(16.4 \%)$ and very rarely make it to the IS in the time interval considered $(0.4 \%)$. Particles that entrain back to the $\mathrm{CC}(5.9 \%)$ were originally detrained. Particles originating in the VS cross over to both the CC and IS, of which $9.7 \%$ entrain back. Entrainment into the $\mathrm{CC}(17.7 \%)$ is much more dominant than detrainment into the IS (5.6\%). The CC-VS mixing is nearly symmetrical, showing nearly equal transport of particles between the two layers. Conversely, VS-IS mixing is highly skewed with particles originating in the IS showing a very high preference to entrain into the VS $(16.3 \%)$ compared to particles starting from the VS detraining into the IS.

Analysis of the particles that originate and finish in the IS $(2.3 \%)$ reveals that these are made up almost entirely (99\%) of particles that entrain and detrain back from the VS. A negligible percentage of particles detrain into the environment and entrain back. The almost one-sided crossing of particles at the TNTI is also revealed by the high percentage of particles $(21.4 \%)$ that originate from the environment and cross into the IS and the negligible percentage moving in the opposite direction. Since the shell thickness is increasing linearly (NHvR20), we can consider the TNTI as a moving interface that would uniformly entrain particles resident in the environment, i.e., $\Delta N_{e}=n A \Delta h$. Here $\Delta N_{e}$ is the number of particles entrained when the interface moves a horizontal distance $\Delta h$ covering an area $A$, and $n=N_{p} / V$ is the particle number density, where $V$ is the domain volume where particles are seeded. Therefore, the number of particles entrained over a time step $d t$ is given by $d N_{e} / d t=n A d h / d t$. A comparison between the number of particles entraining across the TNTI calculated directly from the DNS data, and from the model show a very good agreement (not shown). A moving interface results in a constant number of particles entrained from the environment in our setup with negligible detrainment across 
the TNTI. This explains the skewness in crossings at the TNTI. Yeo and Romps (2012) have shown that a high percentage of air entrained at the cloud boundary had previously been detrained from the cloud. However, entrainment at the TNTI in our setup is almost entirely from the environment. A similar argument can be used to explain the skewness at the VSB. Since the VSB is moving as a function of time as the visible shell thickens, the entrainment from the IS to the VS dominates the detrainment from VS to IS.

Another striking result from Fig. 5 is that a negligible percentage of particles travel all the way from the $\mathrm{CC}$ to the environment and vice versa. This will be addressed further in section 6 where we investigate the degree of premixing done by the shell and how it acts as a buffer layer between the CC and the environment.

A better perspective of the path followed by the particles can be obtained by looking at the average times taken by particles to travel across different zones. This quantity is determined by calculating how long a particle resides in a zone after entering it via an interface. The residence times normalized by the shell time scale $\tau$ are shown in Fig. 6. Calculating the average time after seeding to cross the nearest interface from each zone is quite deceptive since this is highly dependent on the seeding density. We will hence avoid this calculation and focus on residence times after entrainment or detrainment across an interface. For particles originating in the $\mathrm{CC}$, the nearest interface will be the $\mathrm{CCB}$, while for those originating in the VS, the CCB and the VSB are the nearest interfaces. For particles from the IS, the VSB and the TNTI are the nearest interfaces and for those from the environment, the TNTI is the sole interface of interest.

Particles originating in the $\mathrm{CC}$ have a mean residence time of $0.44 \tau$ in the VS after crossing the CCB and before they detrain into the IS. However, it should be noted that the mean times are dependent on the time duration over which the particle data are collected (12 $\mathrm{s}$ in this simulation). Particles residing for a longer period (greater than $12 \mathrm{~s}$ ) are not included, which could result in a higher magnitude of the mean residence time. Since very few particles in the IS cross the TNTI, the residence time in the IS is not calculated.

A majority of the particles originating from the IS cross the VSB into the VS, where they reside for $0.29 \tau$ before crossing into the CC. Particles originating in the environment cross the TNTI and remain in the IS for $0.46 \tau$. A small percentage (5\%) cross over to the VS and reside there for $0.55 \tau$.

\section{Entrainment at the cloud edge}

In this section we analyze the time histories of particles that cross the three interfaces. This will reveal particle behavior before, at and after crossing each interface, and is information that is impossible to obtain with Eulerian statistics. For this analysis, we consider each interface individually and look at all particle crossings across it. A particle is considered to have entrained when it moves to a different zone from right to left (toward the $\mathrm{CC}$ ) and to have detrained when it moves to a different zone from left to right (toward the environment). The time variable $t_{e}$ and $t_{d}$ represent the time at which a

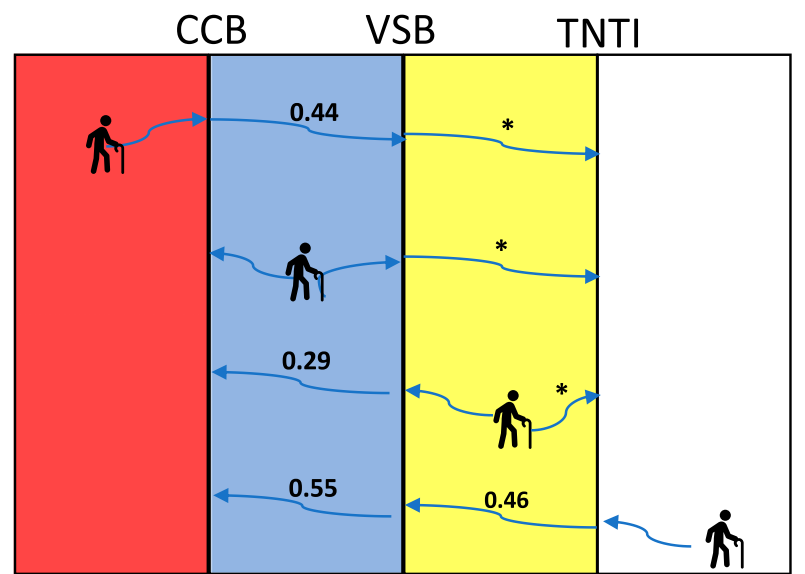

FIG. 6. Average time taken by all entraining/detraining particles to travel across a zone. The values shown are averages of the residence times in a zone normalized with $\tau$. Asterisk indicates insufficient number of particles traveling from interface to interface to get a reliable mean value.

particle entrains or detrains across an interface, respectively. In case of multiple entrainment events by the same particle across a particular interface, the time of occurrence of the first entrainment event is taken as the entrainment time $t_{e}$. For detrainment we follow a slightly different procedure. A detrainment event is considered to take place only if a particle moves to a different zone and resides there, i.e., detraining particles that are entrained back are not considered in the analysis while particles that detrain-entrain-detrain are counted. In short, for a particle to be considered in the detrainment analysis, the last crossing between zones should have been from right to left. The time of the last crossing from right to left is taken as the detrainment time $t_{d}$. We adopted such an approach since entraining particles show a tendency to detrain after one or two time steps immediately after entrainment, before entraining again at the next time step. Counting such short term detrainment events contaminates the plot and are hence these are not considered in the detrainment plot.

Figure 7 shows boxplots of the enstrophy $\omega^{2}$, and buoyancy $b$ of all entraining particles. In boxplots, groups of particle data are expressed using their quartiles. The tops and bottoms of the box are the first and third quartiles (or the 25th and 75th percentiles), and the red line is the median of the particle data group. The $x$ axis represents the time before and after a crossing and is normalized with the shell time scale $\tau$. The whiskers (showing variability from the first and third quartiles) and outlier points for the boxplot have been removed from the figure for the sake of clarity. A sharp jump in the enstrophy values is observed as particles cross the TNTI as shown in Fig. 7a. This is consistent with vorticity jumps that are observed across the TNTI in several flows (da Silva et al. 2014). Once entrained into the IS, the particles acquire enstrophy rapidly. The buoyancy shows a very smooth decrease for particles entraining across the TNTI as shown in Fig. 7b. As shown in section 2 , the outer boundary of the (invisible) shell where the buoyancy drops to zero coincides with the TNTI. The value of 
(a)

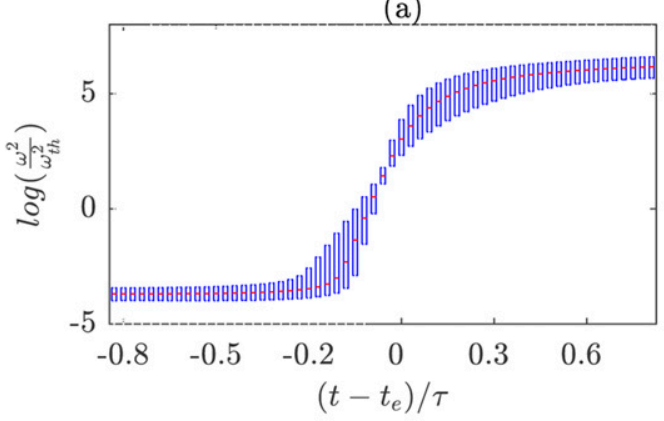

(c)

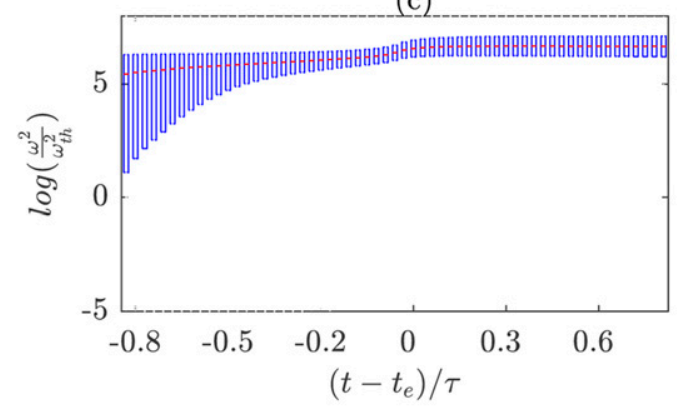

(e)

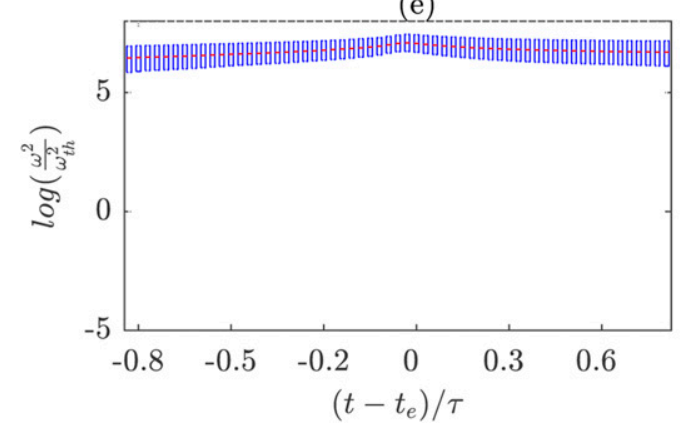

(b)

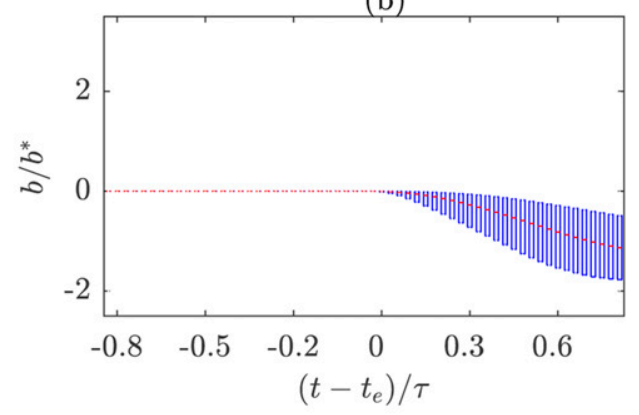

(d)

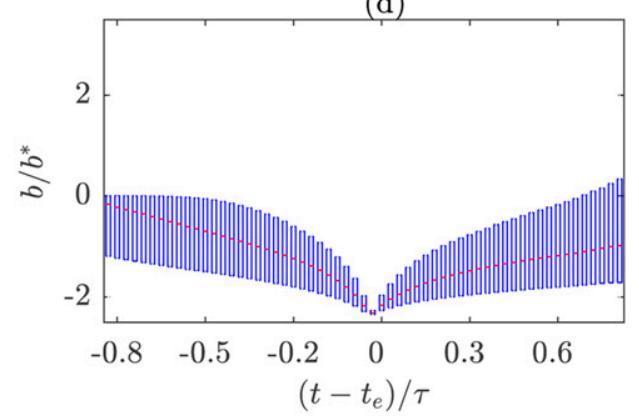

(f)

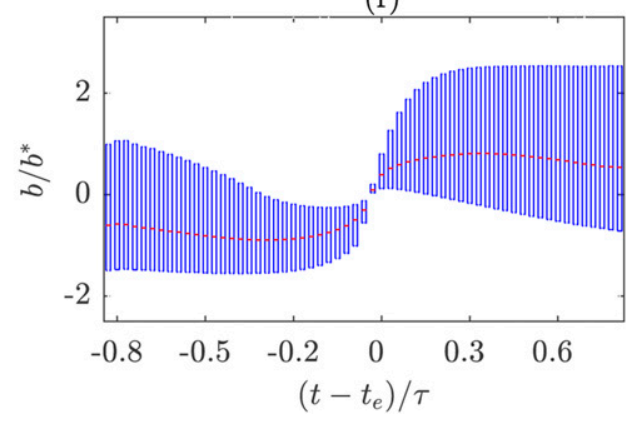

FIG. 7. Boxplot showing the time histories for (left) $\omega^{2}$ and (right) $b$ for entraining particles crossing the (a),(b) TNTI, (c),(d) VSB, and (e),(f) CCB. Outlier points and whiskers for extreme values have been removed for the sake of clarity.

buoyancy is zero at the environment and since almost all the particles crossing the TNTI originate from the environment (as was also shown in section 4) there is a smooth decrease in the buoyancy as particles cross into the IS.

No significant change in the particle enstrophy is observed as they entrain into the VS through the VSB (Fig. 7c). The VSB resides deep in the shell where turbulence intensities are high, and the particles are not subjected to sharp gradients across this interface. The enstrophy values before entrainment predominantly indicate particles originating from the IS and almost none from the environment. In sharp contrast to the enstrophy, there is a kink in the buoyancy as the particles entrain into the VS. This is due to the VSB coinciding with the buoyancy minimum. The majority of the particles crossing into the VS are from the IS as seen by the negative values of buoyancy before entrainment. This is also consistent with the origin-destination matrix in Fig. 5. Positive values for the buoyancy can be observed for $\left(t-t_{e}\right) / \tau>0.6$, which indicates that particles cross over into the CC.

Particles crossing the CCB show a similar behavior as those crossing the VSB with the enstrophy remaining almost the same across the interface. The buoyancy shows a kink at the point where the particles cross over from the negatively buoyant VS into the positively buoyant CC. The positive and negative values for the buoyancy before entrainment indicate that entraining particles include those that originate from the VS as well as those that have been recently detrained from the core. At about $0.3 \tau$ before entrainment, the buoyancy is almost entirely negative indicating only particles from the VS. 
(a)

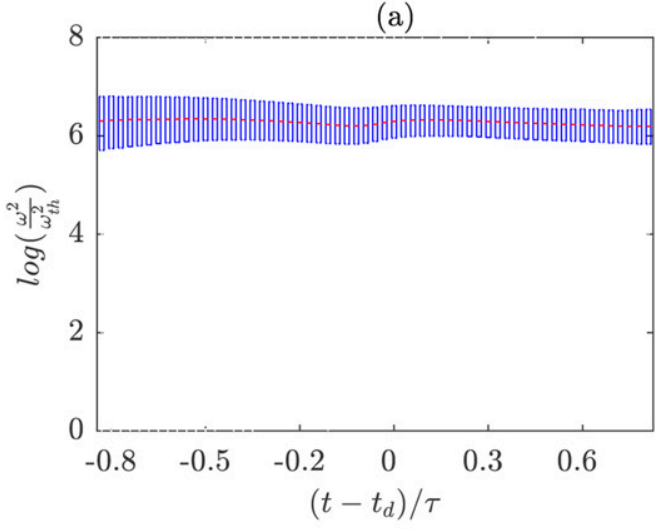

(c)

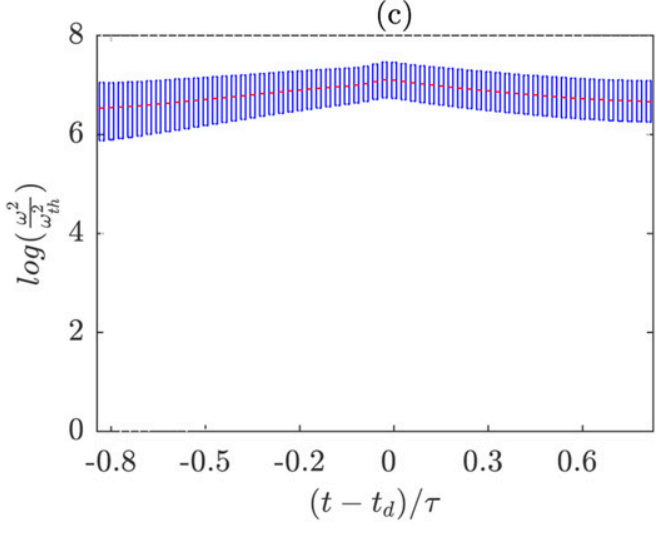

(b)

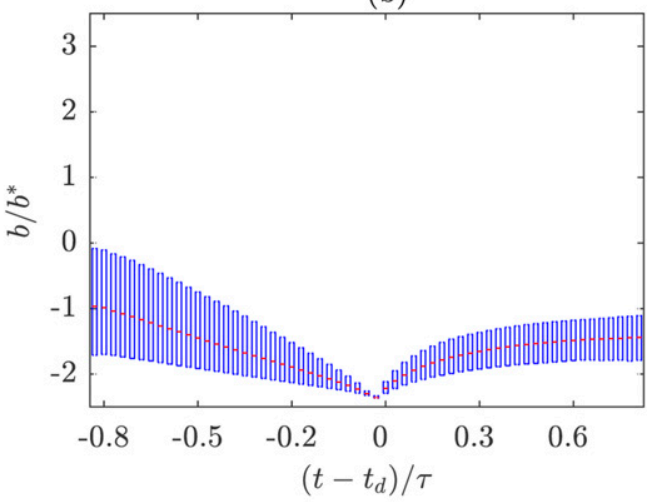

(d)

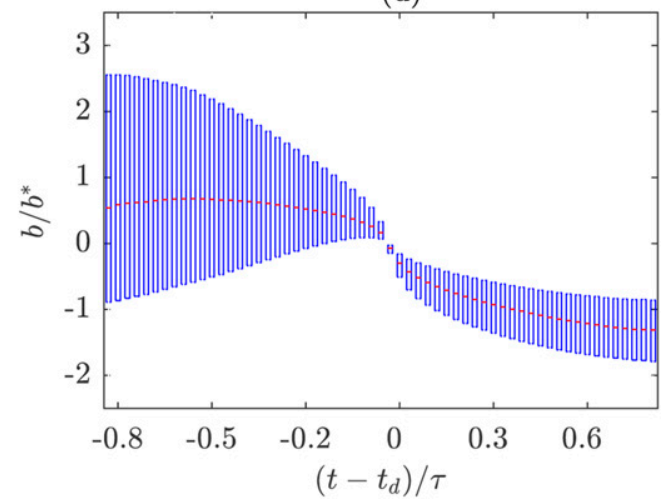

FIG. 8. Boxplot showing the time histories for (left) $\omega^{2}$ and (right) $b$ for detraining particles crossing the (a),(b) VSB and (c),(d) CCB. Outlier points and whiskers for extreme values have been removed for the sake of clarity.

The finite jump in enstrophy values at the TNTI combined with the relatively flat behavior across the VSB and the CCB highlights the relevance of the TNTI as the entraining cloud interface. The VSB and the CCB can be considered to be a crossover point where particle buoyancy decreases significantly (to a minimum at the VSB and zero at the CCB) as a result of evaporative cooling.

Detrainment plots show similar behaviors (Fig. 8). We only show plots for the VSB and the CCB. This is because we observed very few qualifying detrainment events at the TNTI compared to the VSB and the CCB as shown in section 4. This is an indication of the premixing done by the shell. Particles originating from the CC and detraining all the way to the environment is an extremely rare event within the time scale studied $(0.67 \tau)$. Similar to the entrainment plots, there are no finite jumps in enstrophy during detrainment across the VSB and the CCB. Across the VSB, the majority of detraining particles are from the VS (entirely negative buoyancy at start of the plot). But across the CCB, there are particles that originate from both the $\mathrm{CC}$ and the VS. The detraining particles that originate from the $\mathrm{CC}$ are probably those at the edge of the $\mathrm{CC}$ that mix with the negatively buoyant VS as a result of which they lose positive buoyancy and are detrained from the CC. A closer look at the properties of entraining and detraining particles is taken in the next section.

\section{Shell premixing and preferential entrainment at interfaces}

In this section we look at how effective the shell is in premixing the entraining and detraining parcels. All particles crossing the CCB, VSB and the TNTI at normalized time $t^{*}=$ 0.67 are considered irrespective of their origin. As mentioned in the introduction, Dawe and Austin (2011) attributed the difference between the bulk and local entrainment rates to be essentially due to the presence of the subsiding shell around the CC. This means that the properties of entraining and detraining air can no longer be the same as the mean values in the environment and the CC, respectively. This is explored in Fig. 9, which shows histograms of the total water specific humidity $q_{t}$ (Figs. 9a,c,e) and vertical velocity $w$ (Figs. 9b,d,f) for all particles that have entrained and detrained across the interfaces. Figure 9 shows entrainment and detrainment across the CCB (Figs. 9a,b), the VSB (Figs. 9c,d), and the TNTI (Figs. 9e,f).

The histograms in Figs. 9a and 9b are clearly skewed and suggest that the properties (vertical velocity $w$ and total water specific humidity $q_{t}$ ) of the entraining and detraining particles 
(a) CC - VS

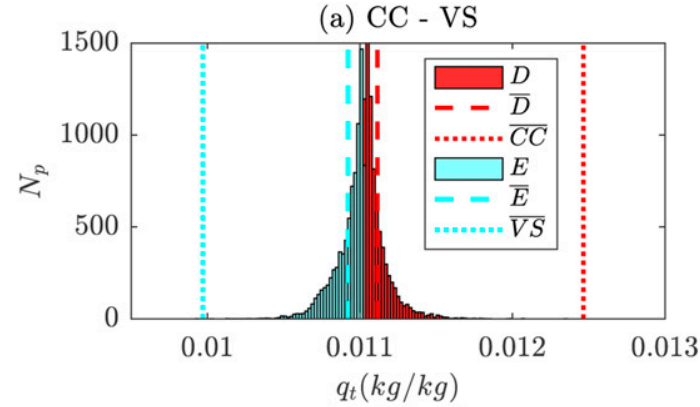

(c) VS - IS

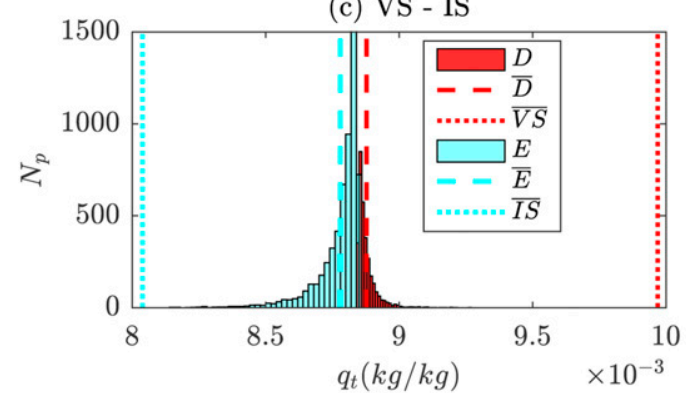

(e) IS - Env

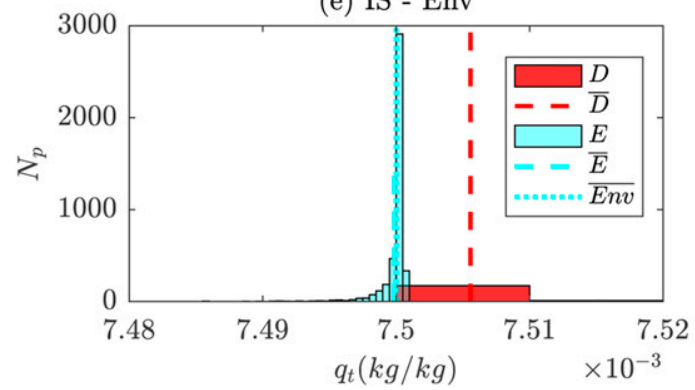

(b) $\mathrm{CC}$ - VS

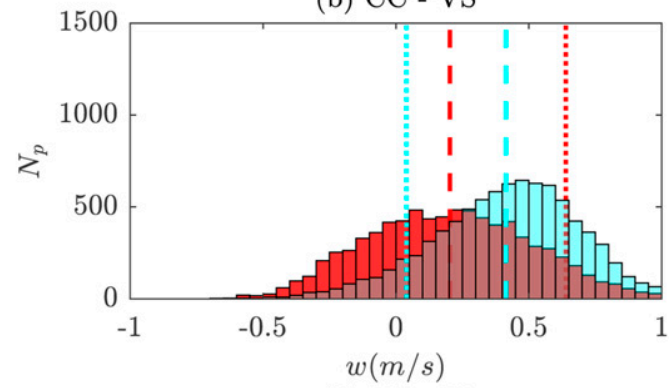

(d) VS - IS

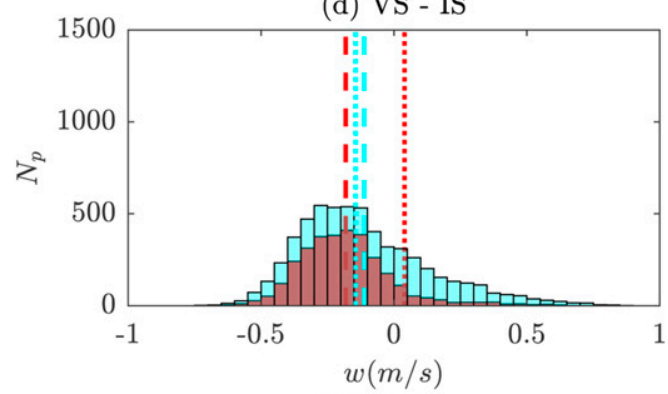

(f) IS - Env

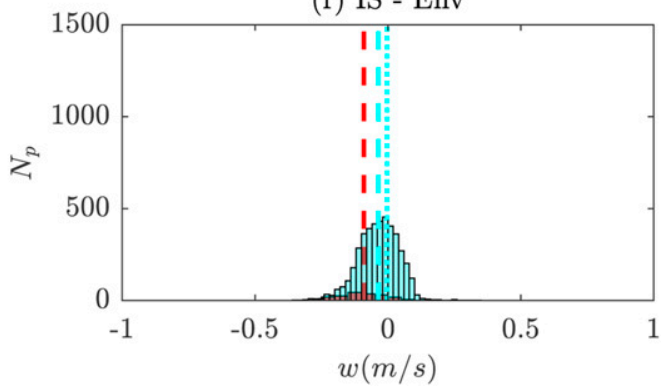

FIG. 9. Histograms showing the properties of particles that have E-entrained (cyan) and D-detrained (red) across the (a),(b) CCB, (c),(d) VSB, and (e),(f) the TNTI at time $t^{*}=0.67$. The properties shown are (a),(c),(e) $q_{t}$ and (b),(d),(f) $w$. The cyan dashed and dot-dashed lines indicate mean magnitudes of the entrained particles and all particles in the entraining zone, respectively, and the red dashed and dotted lines indicate the mean of detraining particles and all the particles in the detraining zone.

do not have a mean value equal to the horizontal slab average values of the environment and $\mathrm{CC}$, respectively. The particles entraining (cyan) across the CCB have a mean value (cyan dashed line) of $w$ and $q_{t}$ closer to those at the inner edge of the VS. The mean of $w$ and $q_{t}$ is also higher than the mean for all particles in the VS (cyan dotted line), i.e., there is clear evidence for preferential entrainment of particles, which have a $q_{t}$ and $w$ higher than the mean values in the VS. Dawe and Austin (2011) explained this preferential entrainment by the presence of negatively buoyant regions, which still had positive vertical velocity and condensed liquid water. As these parcels rise, there is latent heat release due to further condensation, thus making the parcels positively buoyant, which leads to them being entrained into the CC. This is true in the current simulation as well. In the buoyancy phase, even though the mean value of vertical velocity in the shell is negative, there still are upward-moving negatively buoyant parcels with liquid water (especially in the VS). We would not expect to see this effect to happen at the VSB and this is indeed true as seen in
Figs. 9c and 9d. However, the mean values of $q_{t}$ (cyan dashed line) and $\theta_{l}$ (not shown) of particles crossing into the VS coincides with the mean saturation value $\overline{q_{s}}$ and $\bar{\theta}_{s}{ }^{1}$. This again highlights the degree to which the shell premixes the entraining air. Entrainment across the TNTI is shown in Figs. 9e and 9f and involves particles only from the environment as was shown in section 4. The mean value of $q_{t}$ of particles entraining into the IS coincides with the mean of the Environment as expected. Entraining particles also have a mean vertical velocity

\footnotetext{
${ }^{1}$ Considering a classical $q_{t}-\theta_{l}$ mixing diagram and assuming linear mixing between a saturated cloud and unsaturated environment parcel, the intermediate thermodynamic states of the cloud environment mixture can be assumed to lie on a straight line connecting the two initial states. This means that the mean properties of the saturation mixture, $\overline{q_{s}}$ and $\bar{\theta}_{s}$, are the coordinates of the point where the mixing line crosses the saturation curve (Fig. $2 \mathrm{a}$ in NHvR20).
} 


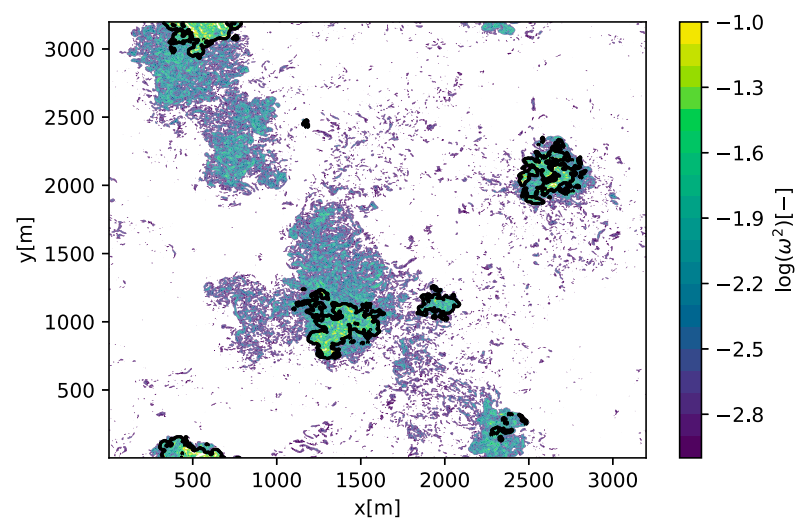

FIG. 10. Contour plot of enstrophy at a midcloud layer $(z=$ $1000 \mathrm{~m})$. Black contours denote the cloud boundary.

that is slightly negative. A possible reason for this could be the rapid entrainment of particles that were detrained from the IS and have negative velocities. Figure 9 also shows the properties of particles detraining across the CCB and the VSB (red). Figure 9a clearly shows that it is the drier air at the edges of the $\mathrm{CC}$ that is detraining across the CCB. For the $\mathrm{CC}$, $\overline{q_{t}}=12.5 \mathrm{~g} \mathrm{~kg}^{-1}$ (red dotted line) while the mean of the detraining particles is significantly lower at around $11.1 \mathrm{~g} \mathrm{~kg}^{-1}$ (red dashed line). Another interesting observation is that the mean vertical velocity of the detraining parcels is positive as shown in Fig. 9b. These could be the parcels that are negatively buoyant at the edges of the $\mathrm{CC}$ but still moving up due to the positive vertical momentum of the fluid in the $\mathrm{CC}$. The vertical velocity histogram also shows a very similar behavior to the entrainment. Detrainment across the VSB (Figs. 9c,d) shows a very small sample range for $q_{t}$. Very few particles detrain into the IS, which is consistent with the percentages seen in the matrix in Fig. 5. The mean of the vertical velocity of the detraining particles is negative and is very close to that of the IS (blue dotted line). Detrainment across the TNTI is almost entirely negligible with very few particles crossing from the IS.

\section{Presence of the interface in LES}

The DNS setup is highly idealized. To verify whether the results for the DNS extrapolate to situations in which the full cloud life cycle is represented, we ran a high resolution LES of shear-free BOMEX case (Holland and Rasmusson 1973) at a resolution of $4.1 \mathrm{~m}$. Figure 10 shows a contour plot of the horizontal cross-section of enstrophy at a midcloud layer $(z=$ $1000 \mathrm{~m}$ ). A visual inspection suggests that most of the IS is indeed localized, and usually centered around the clouds (denoted in black contours). Typical values of enstrophy drop from $10^{-1}$ in the clouds to below $10^{-3}$ in the environment. Figure 11 shows the mass flux density (Fig. 11a) and enstrophy (Fig. 11b) plotted as a function of the distance to the nearest cloud edge. The plots clearly show that the shell itself is confined to within less than $200 \mathrm{~m}$ from the nearest cloud edge. The additional empty IS areas at plot locations $(600,2200) \mathrm{m}$ and $(1300,1500) \mathrm{m}$, are likely related to a recently dissipated cloud and do not necessarily contribute to the negative mass flux. Clouds will also generate internal waves, which in turn produce vorticity in the environment (Fodor and Mellado 2020). However, detangling both is subtle and is beyond the scope of this work.

Figure 12 shows the conditionally sampled enstrophy over the cloud layer, with each layer defined similar to the definitions in section 3 , but with the adjusted thresholds $q_{l, \text { th }}=0$ and $\omega_{\mathrm{th}}^{2}=10^{-3} \mathrm{~s}^{-2}$. For these and the consecutive graphs, we have used the LES output between the third and tenth hour, with a sample time of $0.5 \mathrm{~h}$. The IS is defined with $\omega_{\mathrm{th}}^{2}=10^{-3} \mathrm{~s}^{-2}$ and with no threshold on the buoyancy. Throughout the cloud layer, the enstrophy in the cloud and its immediate surroundings is an order of magnitude higher than the enstrophy in the environment. This enstrophy jump across the two zones shows that similar to the TNTI observed in the DNS, we can observe a clear interface between the IS and the environment as well. Because the ambient fluid is still turbulent, this is now actually a turbulent-turbulent interface (TTI). According to Kankanwadi and Buxton (2020), the adjustment in enstrophy across the TTI is analogous to the TNTI. (a)

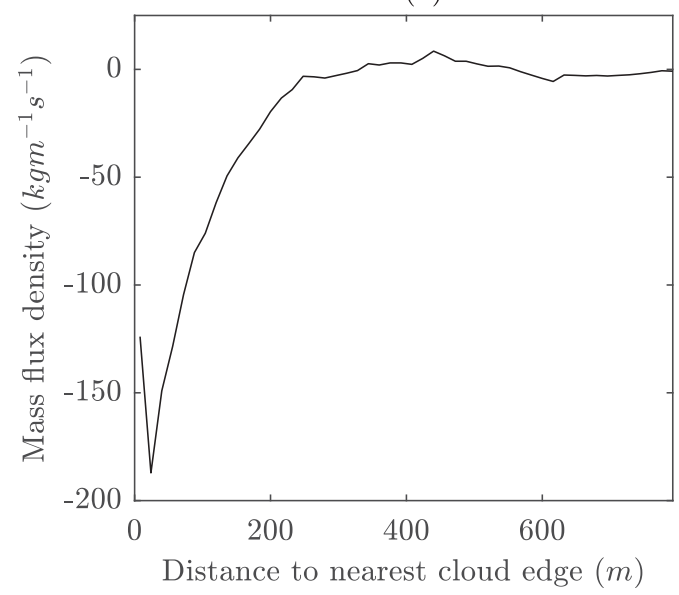

(b)

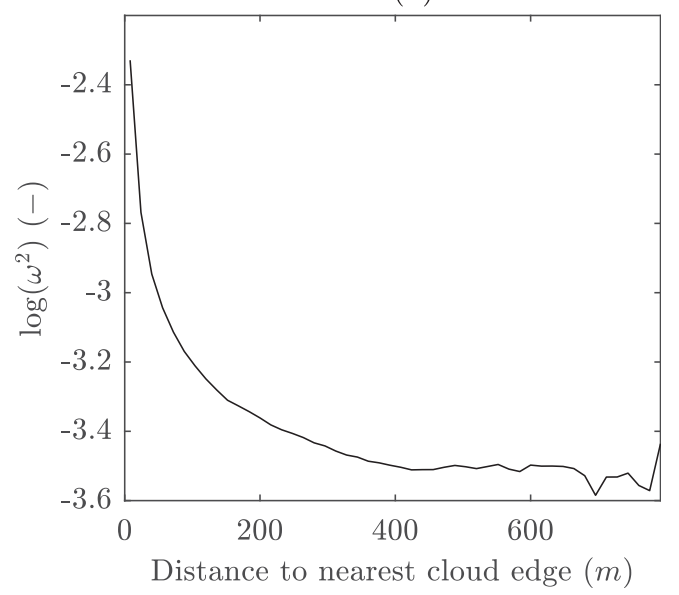

FIG. 11. (a) Mass flux density and (b) enstrophy as a function of the distance to the nearest cloud edge. 


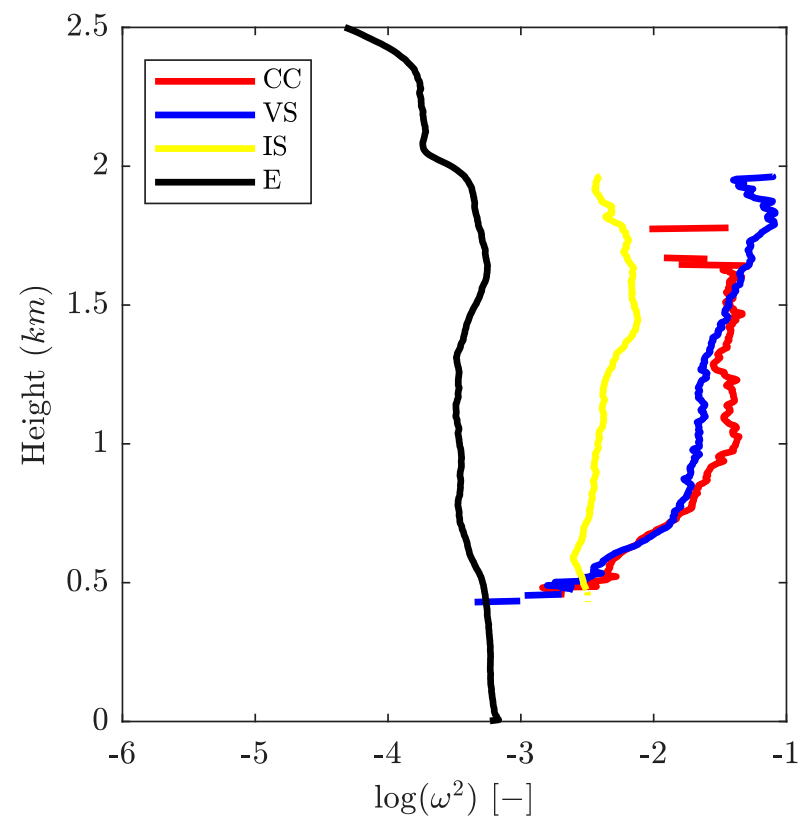

FIG. 12. Conditionally sampled enstrophy in each of the zones, averaged between hours 4 and 10 of the simulation, with a sample time of $0.5 \mathrm{~h}$. Red: cloud core; blue: visible shell; yellow: invisible shell; black: environment.

The choice of the enstrophy threshold $\omega_{\text {th }}^{2}=10^{-3} \mathrm{~s}^{-2}$ can be justified by looking at the cumulative mass flux for all noncloudy grid cells plotted against the enstrophy as shown in Fig. 13. Starting from zero at high enstrophy (clouds), there is a small bump (perhaps evaporating cloud tops), after which a steep decrease in mass flux and an inflection point at $\omega^{2}=$ $10^{-3} \mathrm{~s}^{-2}$ is seen. In other words, the mass flux per unit enstrophy is the highest in the IS, which is centered around the clouds (see Fig. 12). Finally, Fig. 14 shows the conditionally sampled mass flux profile. In the middle of the cloud layer, far away from the lifting condensation level and the level of neutral buoyancy, the IS is responsible for the majority of the negative mass flux, which remains almost constant from the cloud base at $0.5 \mathrm{~km}$ up to $1.1 \mathrm{~km}$. Highly negative values of mass flux in the environment at around $1.2-1.5 \mathrm{~km}$ are observed. One possible reason could be that the cloud top generates internal waves with a net negative mass flux because the initial upward motion is still part of the high enstrophy region. However, between the height range of $0.75-1.1 \mathrm{~km}$, the mass flux in the environment is close to zero. Overall, the results suggest that, in the mid layer of the cloud (for the conditions considered in this simulation), the upward mass flux in the cloud core is balanced by the negative mass flux in the IS with no lateral mass exchange at the TTI, or the net exchange rate across the TTI is zero. This is consistent with the results in Jonker et al. (2008) where the mass flux in the shell (within $200 \mathrm{~m}$ of the cloud edge) was shown to compensate for about $80 \%$ of the in-cloud mass flux.

Our DNS study can be considered to correspond to the LES results between 0.5 and $0.8 \mathrm{~km}$. In this region, the gradient of

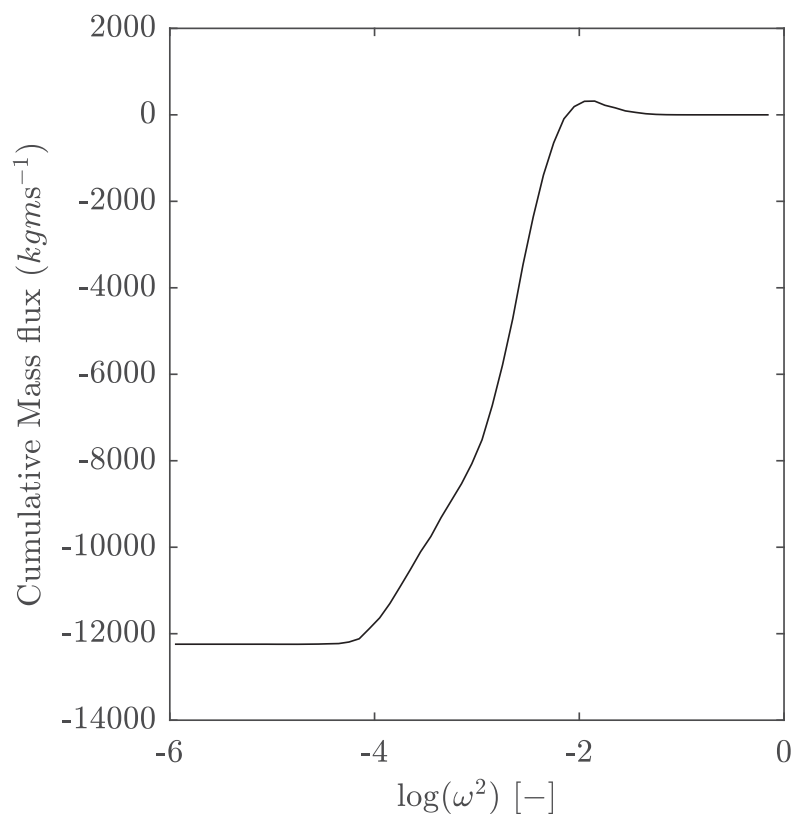

FIG. 13. Cumulative mass flux vs enstrophy for noncloudy regions, averaged between hours 4 and 10 of the simulation, with a sample time of $0.5 \mathrm{~h}$.

the mass flux in the environment shows a negative slope, which represents air being entrained from the environment. The DNS study reveals a similar net entrainment at the TNTI as mentioned in section 4 .

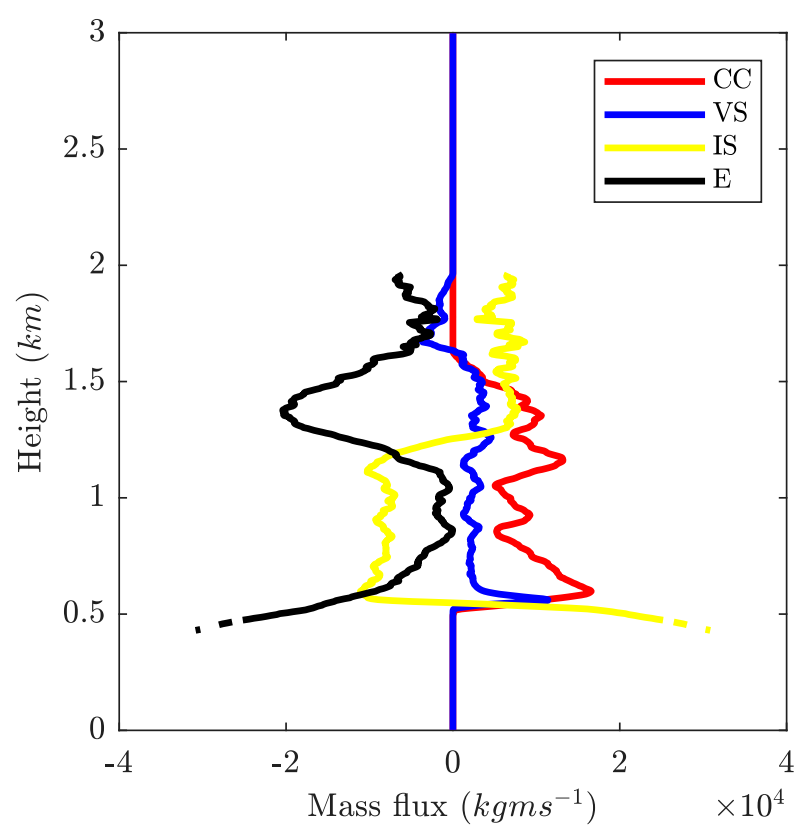

FIG. 14. Conditionally sampled mass flux in each zone, averaged between hours 4 and 10 of the simulation, with a sample time of $0.5 \mathrm{~h}$. Red: cloud core; blue: visible shell; yellow: invisible shell; black: environment. 


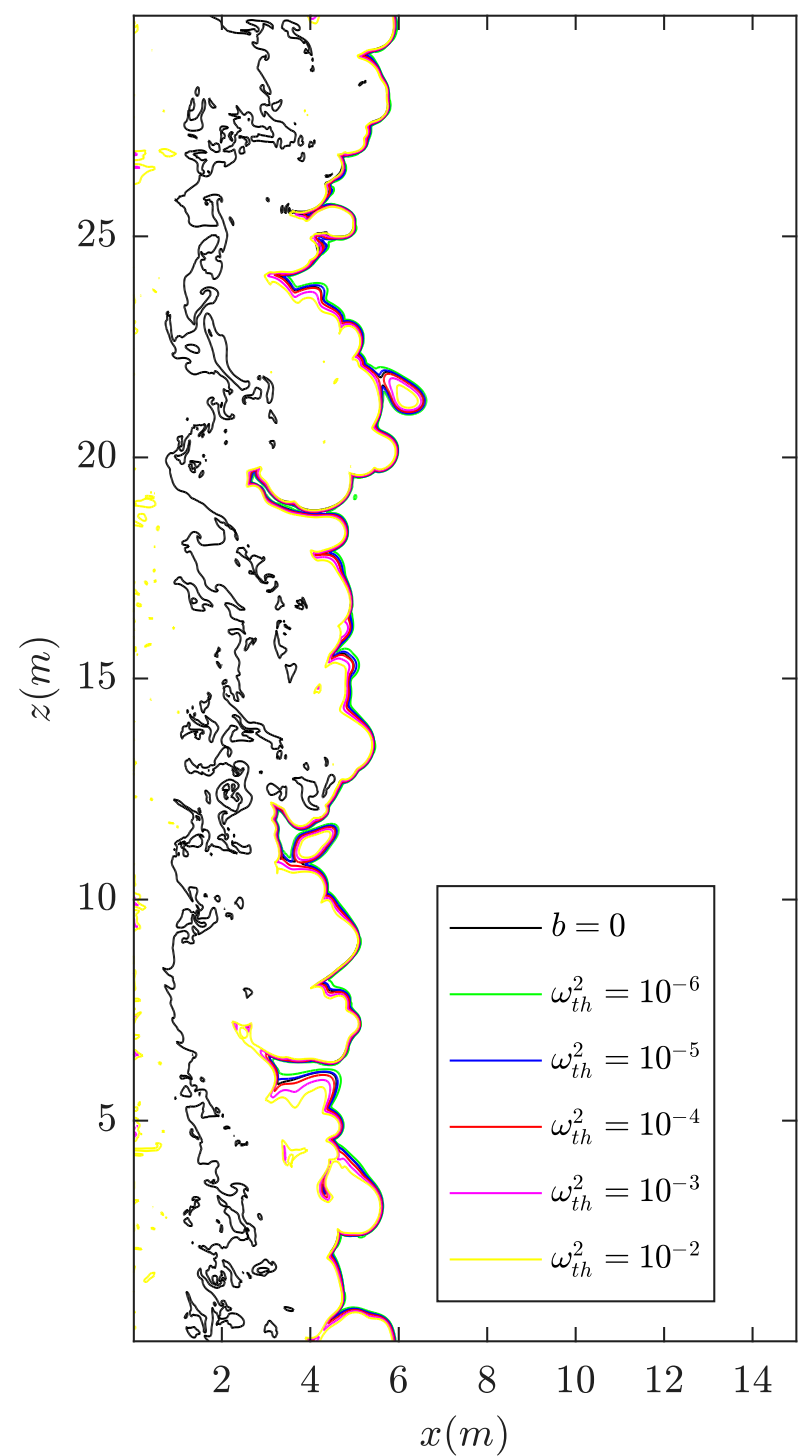

FIG. A1. Isolines of $b=0$ (black) at the time of seeding $\left(t_{0}\right)$. Superimposed are the different interfaces obtained by applying different threshold values to the enstrophy field $\omega_{\text {th }}^{2}$.

\section{Discussion and concluding remarks}

A numerical study of the different interfaces at the edge of a cumulus cloud was performed using DNS and LES. The DNS study reveals the presence of four distinct zones that can be detected by applying thresholds on the enstrophy $\omega^{2}$ and the specific humidity of liquid water $q_{l}$. The four zones have been defined as the cloud core, the visible and invisible shells, and the environment layer. The different zones give rise to three distinct interfaces: the cloud core boundary, visible shell boundary (traditionally considered the cloud edge in parameterization studies), and a turbulent-nonturbulent interface. Massless Lagrangian particles were introduced at the cloud edge and their trajectories and properties were tracked.

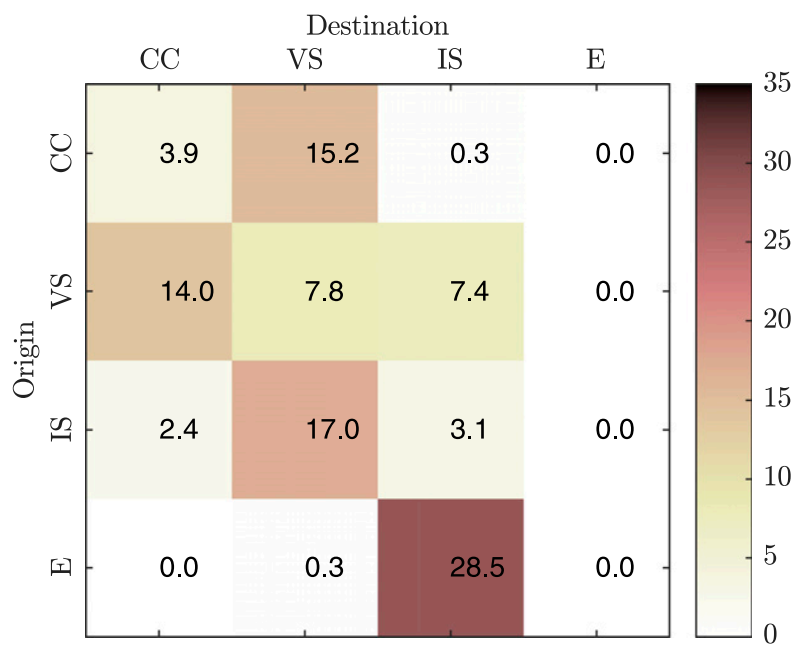

FIG. B1. Origin-destination matrix for particle final locations showing percentage-wise distribution of particles based on zone of origin at the time of seeding and the final destination zone at $t^{*}=0.99$.

One of the main findings of this work is the detection of an interface (in both the DNS and LES studies) between the invisible shell and the environment, separating regions of different turbulence intensities and across which a finite jump in enstrophy is observed. This layer extends beyond the traditional cloud boundary defined by the liquid water specific humidity. For the DNS, the environment is quiescent and the interface is representative of the classical turbulent-nonturbulent interface (da Silva et al. 2014). The LES studies, which are substantially more complex and do not have a quiescent environment, reveal an interface separating zones with distinctly different levels of enstrophy, and are hence more appropriately described by a turbulent-turbulent interface, with the majority of the downward mass flux contained within this interface. Pertinently, the DNS results indicate there is no dynamic distinction between the visible shell and the invisible shell, indicating the two are part of the same system. It remains to be seen whether the IS plays a dynamical role in the evolution of cloud boundaries, but the current study clearly indicates that the cloud edge extends beyond the visible shell.

The DNS results reveal that Lagrangian particles experience finite jumps in its enstrophy and a smooth increase in buoyancy when it crosses the TNTI. The traditional cloud boundary considered in LES entrainment studies, the visible shell boundary, is essentially a crossover station, which does not significantly affect entraining or detraining parcels. It coincides with the location of minimum buoyancy within the shell and parcels entraining across this boundary have saturation values that can be predicted from the mixing diagram.

We also observe preferential entrainment across the $\mathrm{CCB}$ as shown by Dawe and Austin (2011), where particles with total humidity $q_{t}$ and vertical velocity $w$ higher than the mean of all parcels in the shell are more likely to entrain. Also, drier air that is close to the CCB is more likely to detrain. This drier air 
(a)

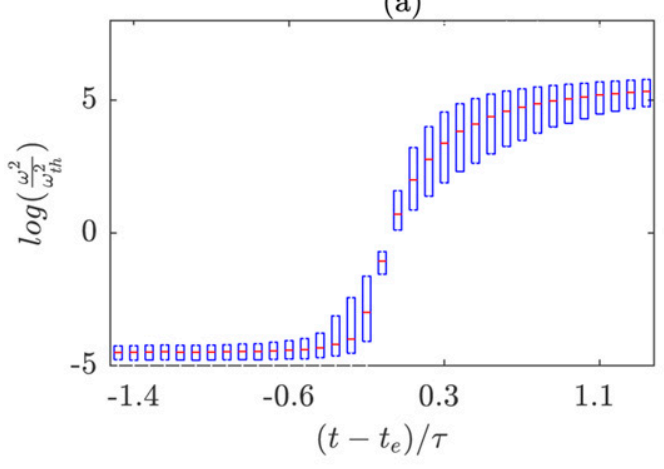

(c)

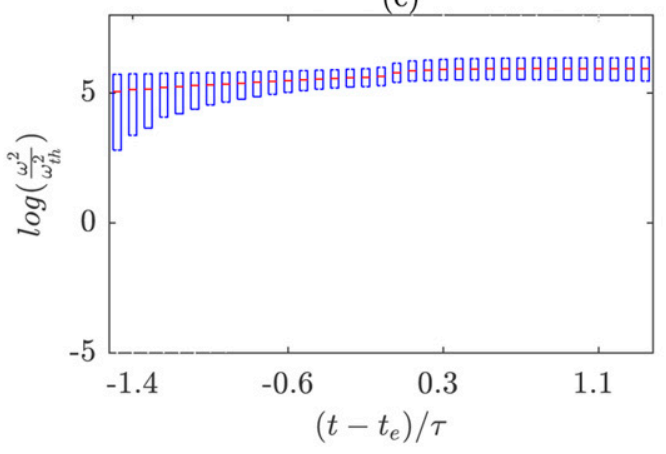

(e)

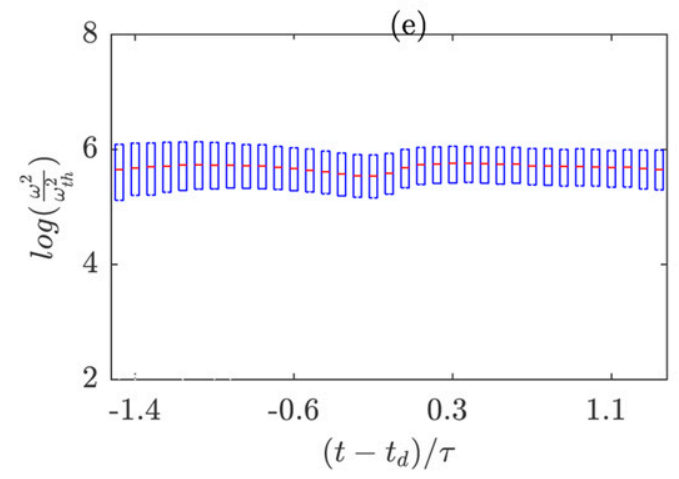

(b)

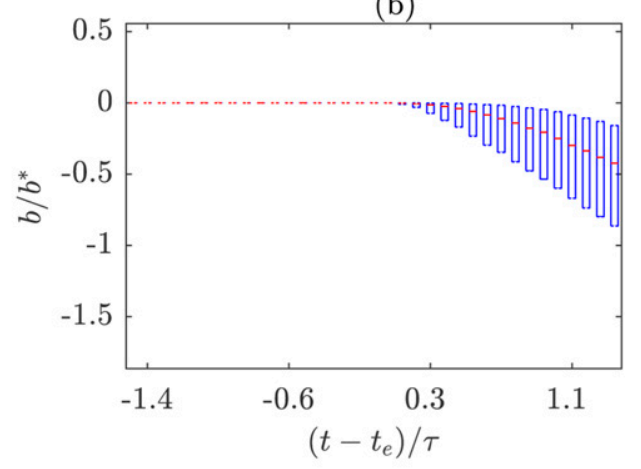

(d)

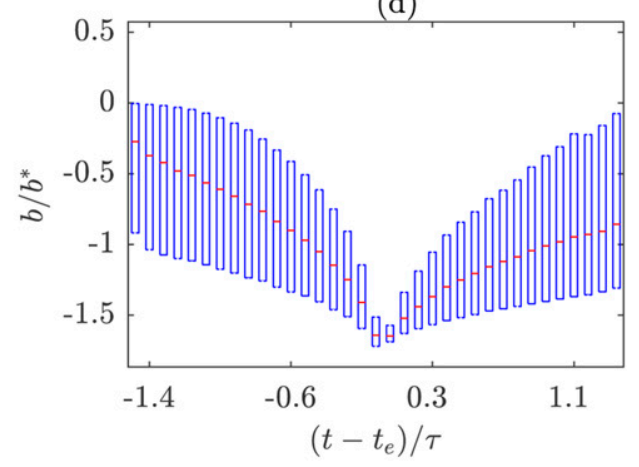

(f)

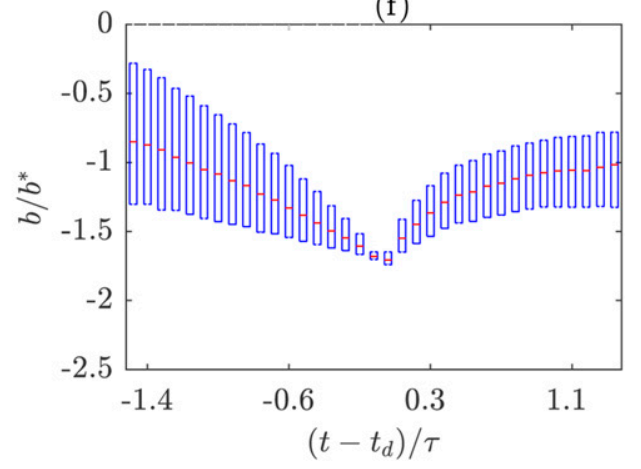

FIG. B2. Time histories for (left) $\omega^{2}$ and (right) $b$ for entraining particles crossing the (a),(b) TNTI and (c),(d) VSB, and for detraining particles crossing (e),(f) the CCB.

is also likely to be negatively buoyant but moving with a positive vertical velocity in the cloud core.

The DNS study is performed using a highly idealized setup. The simplifications include an infinitely long cloud interface without stratification of the environment and no other sources of turbulence such as wind shear. It is also important to highlight the fact that by imposing a periodic boundary condition on a domain size of $30 \mathrm{~m}$, larger eddies in the cloud are suppressed. However, the LESs are performed using a more realistic setup and was able to detect a TTI using the same thresholds as in the DNS, which shows that it is possible to extend the notion of a TNTI to turbulent environments. Indeed, da Silva et al. (2014) describes the interface layer as a thin region with a finite thickness that separates either (i) regions with different turbulent intensity or (ii) turbulent and (external) irrotational flow regions. Conditionally averaged mass flux in the different zones in the LES results reveal that within the mid cloud layer, there is no net exchange across the TTI. The upward mass flux in the cloud core is compensated by the mass flux in the IS with negligible mass flux in the environment.

While we do not observe any detrainment across the TNTI, this does not mean that no air can be transported from the cloud into the environment. The cloud will eventually dissipate, and so will the IS. During this process, the humid air of the cloud will moisten the environment. However, this is an entirely different mechanism than any direct mixing between 
the cloud and the environment, with different results. For instance, if detrained air remains close to the cloud boundary, subsequent entrainment events will do less to dilute the cloud, resulting in stronger updrafts. We also speculate that a slowly dissipating IS may allow for preconditioning certain regions for subsequent convection; further research is needed to confirm or falsify such speculation.

Acknowledgments. Vishnu Nair and Maarten van Reeuwijk acknowledge funding from the Marie-Sklodowska Curie Actions under the European Union's Horizon 2020 research and innovation programme (Grant 675675). Computational resources on the U.K. supercomputing facility ARCHER via the U.K. Turbulence Consortium (EP/R029326/1) and the Imperial College HPC services are gratefully acknowledged. Thijs Heus was supported by the U.S. Department of Energy's Atmospheric System Research, an Office of Science, Office of Biological and Environmental Research program, under Grant DE-SC0017999.

Data availability statement. The DNS data created during this study are openly available from the Zenodo data repository at http://doi.org/10.5281/zenodo.5040419. The LES data are available on request.

\section{APPENDIX A}

\section{Sensitivity to Threshold Values}

A very popular method used to detect a TNTI is by applying a threshold on enstrophy, which allows one to separate the turbulent region from the approximately irrotational, nonturbulent region (da Silva et al. 2014). This value can be selected from a range since statistics have been shown to be insensitive to the exact threshold value (Bisset et al. 2002). We perform an analysis to verify if the location of the TNTI is indeed insensitive to the choice of threshold values in our case setup.

We check a range of magnitudes for $\omega_{\text {th }}^{2}$ from $10^{-3}$ to $10^{-6} \mathrm{~s}^{-2}$. In Fig. A1, the interfaces obtained by applying the different thresholds are plotted. The overlapping interfaces obtained from this range of threshold values point to the fact that these ranges lie in the viscous superlayer observed in interfacial layers and any threshold magnitude within this range can be used as a robust technique to detect the TNTI. Hence, we settle on a value of $\omega_{\text {th }}^{2}=10^{-6} \mathrm{~s}^{-2}$ for this study.

Furthermore, the TNTI can be seen to coincide exactly with the outer boundary of the shell as determined from the isoline of the buoyancy field $b=0$. The inner interface corresponding to the isoline $b=0$ coincides with the $\mathrm{CCB}$.

\section{APPENDIX B}

\section{Sensitivity to Initial Conditions}

The sensitivity to the DNS initial conditions for the different results presented in the paper are analyzed by running a second simulation with different initial parameters. The initial profiles are similar to simulation A06 in NHvR2 20 with $\Delta \theta_{l}=1.8 \mathrm{~K}$, and

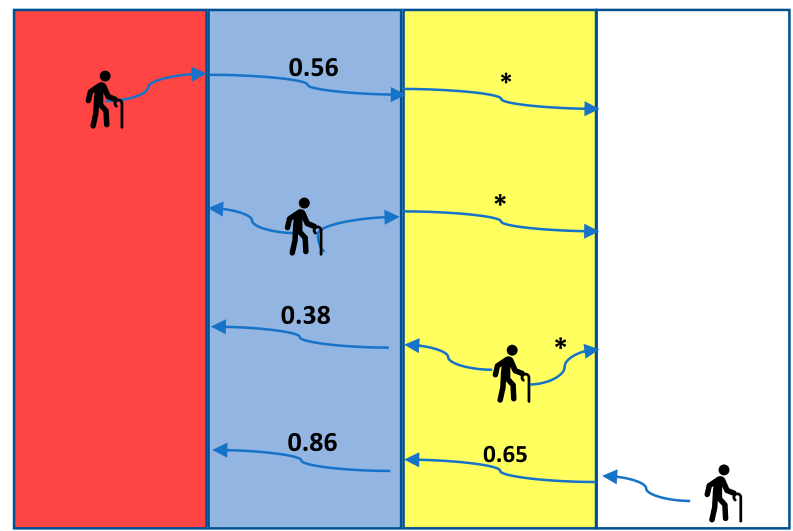

FIG. B3. Average time taken by all entraining/detraining particles to travel across a zone. The values shown are averages of the residence times in a zone normalized with $\tau$. Asterisk indicates insufficient number of particles traveling from interface to interface to get a reliable mean value.

$\Delta q_{t}=1.9 \mathrm{~g} \mathrm{~kg}^{-1}$. A similar domain size and grid resolution is used. The simulation is run for $148 \mathrm{~s}$ and 3 million particles are seeded after $120 \mathrm{~s}$. The Taylor-Reynold's number $\left(\operatorname{Re}_{\lambda}\right)$ at the end of the simulation is 57 . The integral time scale $\tau$ is $24.3 \mathrm{~s}$ and $t_{\eta}$ is $1.04 \mathrm{~s}$.

The origin-destination matrix is plotted in Fig. B1. All the results shown for the main simulation hold for this case as well: symmetric mixing between the CC and the VS, highly skewed mixing between the VS and the IS, and an almost negligible number of particles originating from the environment (CC) and making it all the way to the $\mathrm{CC}$ (environment), respectively, in the time interval of approximately $1 \tau$.

Figure B2 shows the behavior of entraining and detraining particles at the different interfaces. Entraining particles exhibit a finite jump in the enstrophy and a smooth increase in buoyancy after crossing the TNTI (Figs. B2a,b). Both entraining and detraining particles do not show a noticeable increase in enstrophy while crossing the VSB (Figs. B2c,e, respectively), with a kink observed in the buoyancy values (Figs. B2d,f).

Figure B3 shows the mean residence time of the particles after they have crossed an interface. The values are slightly higher than the corresponding values for the main simulation considered in the study owing to the higher time duration $(1 \tau)$ for which statistics are taken.

Figures B1-B3 hence confirm that all the results proposed in the manuscript hold for the new simulation as well and the results are insensitive to the DNS initial conditions.

\section{REFERENCES}

Abma, D., T. Heus, and J. Mellado, 2013: Direct numerical simulation of evaporative cooling at the lateral boundary of shallow cumulus clouds. J. Atmos. Sci., 70, 2088-2102, https:// doi.org/10.1175/JAS-D-12-0230.1.

Arakawa, A., and W. H. Schubert, 1974: Interaction of a cumulus cloud ensemble with the large-scale environment, part I. J. Atmos. Sci., 31, 674-701, https://doi.org/10.1175/1520-0469(1974) 031<0674:IOACCE > 2.0.CO;2. 
Bechtold, P., E. Bazile, F. Guichard, P. Mascart, and E. Richard, 2001: A mass-flux convection scheme for regional and global models. Quart. J. Roy. Meteor. Soc., 127, 869-886, https:// doi.org/10.1002/qj.49712757309.

Bisset, D. K., J. C. R. Hunt, and M. M. Rogers, 2002: The turbulent/ non-turbulent interface bounding a far wake. J. Fluid Mech., 451, 383-410, https://doi.org/10.1017/S0022112001006759.

Bony, S., and Coauthors, 2015: The role of convective-scale precipitation downdrafts in cumulus and synoptic-scale interactions. Nat. Geosci., 8, 261-268, https://doi.org/10.1038/ngeo2398.

Couvreux, F., F. Hourdin, and C. Rio, 2010: Resolved versus parametrized boundary-layer plumes. Part I: A parametrizationoriented conditional sampling in large-eddy simulations. Bound.-Layer Meteor., 134, 441-458, https://doi.org/10.1007/ s10546-009-9456-5.

Craske, J., and M. van Reeuwijk, 2015: Energy dispersion in turbulent jets. Part 1. Direct simulation of steady and unsteady jets. J. Fluid Mech., 763, 500-537, https://doi.org/10.1017/ jfm.2014.640.

da Silva, C., J. Hunt, I. Eames, and J. Westerweel, 2014: Interfacial layers between regions of different turbulence intensity. Annu. Rev. Fluid Mech., 46, 567-590, https://doi.org/10.1146/ annurev-fluid-010313-141357.

Dawe, J., and P. Austin, 2011: The influence of the cloud shell on tracer budget measurements of LES cloud entrainment. J. Atmos. Sci., 68, 2909-2920, https://doi.org/10.1175/2011JAS3658.1.

de Roode, S. R., and C. S. Bretherton, 2003: Mass-flux budgets of shallow cumulus clouds. J. Atmos. Sci., 60, 137-151, https:// doi.org/10.1175/1520-0469(2003)060<0137:MFBOSC >2.0.CO;2.

de Rooy, W. C., and Coauthors, 2013: Entrainment and detrainment in cumulus convection: An overview. Quart. J. Roy. Meteor. Soc., 139, 1-19, https://doi.org/10.1002/qj.1959.

Fodor, K., and J. P. Mellado, 2020: New insights into wind shear effects on entrainment in convective boundary layers using conditional analysis. J. Atmos. Sci., 77, 3227-3248, https:// doi.org/10.1175/JAS-D-19-0345.1.

Gerard, L., 2015: Bulk mass-flux perturbation formulation for a unified approach of deep convection at high resolution. Mon. Wea. Rev., 143, 4038-4063, https://doi.org/10.1175/MWR-D15-0030.1.

Gregory, D., and P. R. Rowntree, 1990: A mass flux convection scheme with representation of cloud ensemble characteristics and stability-dependent closure. Mon. Wea. Rev., 118, 1483-1506, https://doi.org/10.1175/1520-0493(1990)118<1483: AMFCSW $>2.0 . \mathrm{CO} ; 2$.

Gu, J.-F., R. S. Plant, C. E. Holloway, T. R. Jones, A. Stirling, P. A. Clark, S. J. Woolnough, and T. L. Webb, 2020: Evaluation of the bulk mass flux formulation using large-eddy simulations. J. Atmos. Sci., 77, 2115-2137, https://doi.org/10.1175/JAS-D-19-0224.1.

Heus, T., and H. Jonker, 2008: Subsiding shells around shallow cumulus clouds. J. Atmos. Sci., 65, 1003-1018, https://doi.org/ 10.1175/2007JAS2322.1.

_- G. van Dijk, H. Jonker, and H. van den Akker, 2008: Mixing in shallow cumulus clouds studied by Lagrangian particle tracking. J. Atmos. Sci., 65, 2581-2597, https://doi.org/10.1175/ 2008JAS2572.1.

Holland, J. Z., and E. M. Rasmusson, 1973: Measurements of the atmospheric mass, energy, and momentum budgets over a 500-kilometer square of tropical ocean. Mon. Wea. Rev., 101, 44-55, https://doi.org/10.1175/1520-0493(1973)101<0044: MOTAME $>2.3 . \mathrm{CO} ; 2$.

Johnson, R. H., 1976: The role of convective-scale precipitation downdrafts in cumulus and synoptic-scale interactions. J. Atmos.
Sci., 33, 1890-1910, https://doi.org/10.1175/1520-0469(1976) $033<1890$ :TROCSP $>2.0$. CO; .

Jonker, H. J., T. Heus, and P. P. Sullivan, 2008: A refined view of vertical mass transport by cumulus convection. Geophys. Res. Lett., 35, L07810, https://doi.org/10.1029/2007GL032606.

Kain, J. S., and J. M. Fritsch, 1990: A one-dimensional entraining/ detraining plume model and its application in convective parameterization. J. Atmos. Sci., 47, 2784-2802, https://doi.org/ 10.1175/1520-0469(1990)047<2784:AODEPM>2.0.CO;2.

Kankanwadi, K. S., and O. R. H. Buxton, 2020: Turbulent entrainment into a cylinder wake from a turbulent background. J. Fluid Mech., 905, A35, https://doi.org/10.1017/ jfm.2020.755.

Moorthi, S., and M. J. Suarez, 1992: Relaxed Arakawa-Schubert: A parameterization of moist convection for general circulation models. Mon. Wea. Rev., 120, 978-1002, https://doi.org/10.1175/ 1520-0493(1992)120<0978:RASAPO > 2.0.CO;2.

Nair, V., T. Heus, and M. van Reeuwijk, 2020: Dynamics of subsiding shells in actively growing clouds with vertical updrafts. J. Atmos. Sci., 77, 1353-1369, https://doi.org/10.1175/JAS-D19-0018.1.

Park, S.-B., P. Gentine, K. Schneider, and M. Farge, 2016: Coherent structures in the boundary and cloud layers: Role of updrafts, subsiding shells, and environmental subsidence. J. Atmos. Sci., 73, 1789-1814, https://doi.org/10.1175/JAS-D-15-0240.1.

- - T. Teus, and P. Gentine, 2017: Role of convective mixing and evaporative cooling in shallow convection. J. Geophys. Res. Atmos., 122, 5351-5363, https://doi.org/10.1002/ 2017JD026466.

Perrin, V., and H. Jonker, 2015: Lagrangian droplet dynamics in the subsiding shell of a cloud using direct numerical simulations. J. Atmos. Sci., 72, 4015-4028, https://doi.org/10.1175/ JAS-D-15-0045.1.

Romps, D., 2010: A direct measure of entrainment. J. Atmos. Sci., 67, 1908-1927, https://doi.org/10.1175/2010JAS3371.1.

Siebesma, A. P., 1998: Shallow cumulus convection. Buoyant Convection in Geophysical Flows, E. J. Plate, Ed., Springer, 441-486.

—_, and J. Cuijpers, 1995: Evaluation of parametric assumptions for shallow cumulus convection. J. Atmos. Sci., 52, 650-666, https://doi.org/10.1175/1520-0469(1995)052<0650: EOPAFS $>2.0 . \mathrm{CO} ; 2$.

- , and Coauthors, 2003: A large eddy simulation intercomparison study of shallow cumulus convection. J. Atmos. Sci., 60, 1201-1219, https://doi.org/10.1175/1520-0469(2003)60<1201: ALESIS > 2.0.CO;2.

Sommeria, G., and J. Deardorff, 1976: Subgrid-scale condensation in models of nonprecipitating clouds. J. Atmos. Sci., 34, 344-355, https://doi.org/10.1175/1520-0469(1977)034<0344: SSCIMO $>2.0 . \mathrm{CO} ; 2$.

Tiedtke, M., 1989: A comprehensive mass flux scheme for cumulus parameterization in large-scale models. Mon. Wea. Rev., 117, 1779-1800, https://doi.org/10.1175/1520-0493(1989)117<1779: ACMFSF $>2.0 . \mathrm{CO} ; 2$.

van Heerwaarden, C. C., B. J. H. van Stratum, T. Heus, J. A. Gibbs, E. Fedorovich, and J. P. Mellado, 2017: MicroHH 1.0: A computational fluid dynamics code for direct numerical simulation and large-eddy simulation of atmospheric boundary layer flows. Geosci. Model Dev., 10, 3145-3165, https://doi.org/ 10.5194/gmd-10-3145-2017.

Yeo, K., and D. M. Romps, 2012: Measurement of convective entrainment using Lagrangian particles. J. Atmos. Sci., 70, 266277, https://doi.org/10.1175/JAS-D-12-0144.1. 